\title{
Explorations of nonperturbative Jackiw-Teitelboim gravity and supergravity
}

\author{
Clifford V. Johnson ${ }^{*}$ \\ Department of Physics and Astronomy, University of Southern California, \\ Los Angeles, California 90089-0484, USA
}

(Received 23 December 2020; accepted 4 January 2021; published 19 February 2021)

\begin{abstract}
Some recently proposed definitions of Jackiw-Teitelboim (JT) gravity and supergravities in terms of combinations of minimal string models are explored, with a focus on physics beyond the perturbative expansion in spacetime topology. While this formally involves solving infinite-order nonlinear differential equations, it is shown that the physics can be extracted to arbitrarily high accuracy in a simple controlled truncation scheme, using a combination of analytical and numerical methods. The nonperturbative spectral densities are explicitly computed and exhibited. The full spectral form factors, involving crucial nonperturbative contributions from wormhole geometries, are also computed and displayed, showing the nonperturbative details of the characteristic "slope," "dip," "ramp," and "plateau" features. It is emphasized that results of this kind can most likely be readily extracted for other types of JT gravity using the same methods.
\end{abstract}

DOI: 10.1103/PhysRevD.103.046013

\section{INTRODUCTION}

There are many reasons to study Jackiw-Teitelboim (JT) gravity $[1,2]$. One of them is the fact that it is a theory of a two-dimensional quantum gravity, where the spacetime is allowed to split and join, changing its topology (characterized by Euler characteristic $\chi=2-2 g-b-c$, where $g$ counts handles, $b$ boundaries, and $c$ crosscaps). In the full theory, the partition function $Z(\beta)$ is a sum over the contributions from all topologies, as well as a nonperturbative part that is not captured by the perturbative expansion in topology:

$$
Z(\beta)=\sum_{\chi} Z_{\chi}(\beta)+\text { nonperturb. }
$$

Here, $Z_{\chi}(\beta)$ stands for the contribution to the partition function from surfaces of Euler characteristic $\chi$. It comes with a factor $e^{\chi S_{0}}$, as $S_{0}$ is a coupling that multiplies the Einstein-Hilbert action in the model. [Although $\chi=1$ for the (leading) disc-order quantities, the subscript 0 will be widely used at leading order henceforth. So the disc level partition function is $Z_{0}$, spectral density is $\rho_{0}$, etc.]

The focus of this paper will be on characterizing the full partition function of the theory, including the full

\footnotetext{
*johnson1@usc.edu
}

Published by the American Physical Society under the terms of the Creative Commons Attribution 4.0 International license. Further distribution of this work must maintain attribution to the author(s) and the published article's title, journal citation, and DOI. Funded by SCOAP ${ }^{3}$. nonperturbative physics, by making explicit aspects of the double-scaled matrix model definitions suggested in Refs. [3,4], which should be considered companion papers to this one. The beautiful work of Refs. [5,6] in defining double-scaled matrix models of (various kinds of) JT gravity is intrinsically perturbative in spirit, since it uses recursion relations connecting different topologies, and the work in Refs. [3,4] is intended as a complementary construction (using minimal strings) that allows more direct access to nonperturbative quantities. The output of this paper will be the first explicit computation of the full spectral densities (and hence the partition functions, by Laplace transform) and explorations of several important phenomena that depend crucially on being able to compute nonperturbative physics.

An example of the latter is the two-point "spectral form factor" shown in Fig. 1, a quantity that helps in diagnosing universal aspects of quantum chaotic behavior $[7,8]$. It was computed using the methods of this paper. This is the first time this quantity (and others like it to be presented later) has been computed fully in JT gravity or supergravity for generic values of $\beta$ and $S_{0}$, and so some time will be spent unpacking the techniques and results. ${ }^{1}$ The late-time "plateau" feature of the curve, and the transition to it from

\footnotetext{
${ }^{1}$ An interesting recent paper [9] presented an expression for the spectral form factor of JT gravity, but in a very special ultralowtemperature scaling limit that allowed a closed form to be written. Also, key aspects of parts of the spectral form factor in special limits were discussed using matrix model techniques in Ref. [10]. In this paper, no special limits on the parameters are taken, and while no closed forms are presented, answers can be systematically extracted for a range of $\beta$ and $S_{0}$.
} 


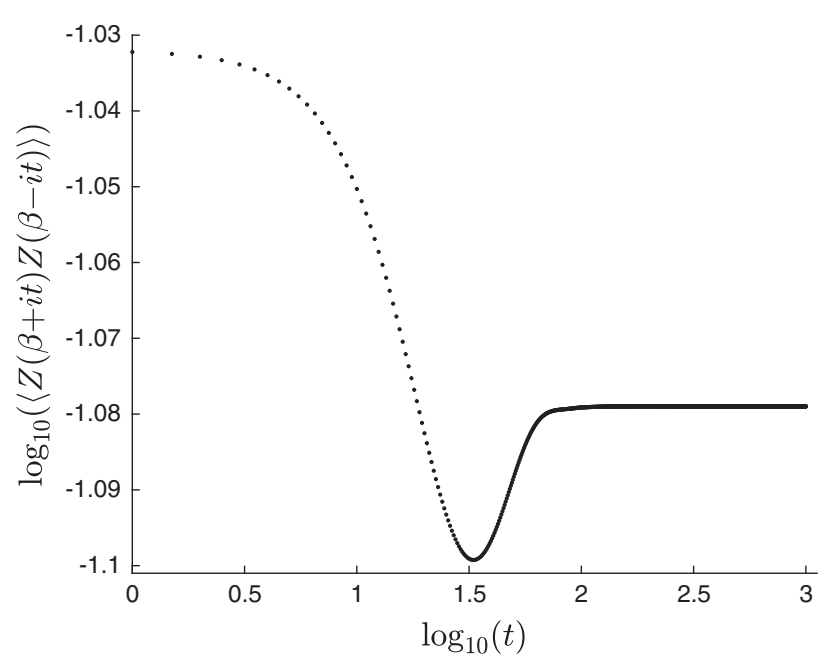

FIG. 1. The full spectral form factor, showing the classic (saxophone) shape made up of a slope, dip, ramp, and plateau. This is computed using the methods of this paper for the $(2,2)$ model of JT supergravity. Here, $\beta=35, \hbar=1 / 5$. (See text.)

the "ramp" behavior, are intrinsically nonperturbative features of wide interest. There are important nonperturbative effects that show up in the slope part too, in some cases, as will be demonstrated. They can sometimes be dramatic, as will be seen in the supergravity examples presented.

Another example of this paper's results is given in Fig. 2. It is the full spectral density (the thicker line, actually made out of data dots) of the model $\rho^{\mathrm{SJT}}(E)$ with the classical result [see Eq. (5)] plotted as a dashed line for comparison. By Laplace transform, this function defines the full nonperturbative partition function for the supergravity theory, and is computed here explicitly for the first time. This JT supergravity model is in fact the $(\boldsymbol{\alpha}, \boldsymbol{\beta})=(2,2)$ matrix

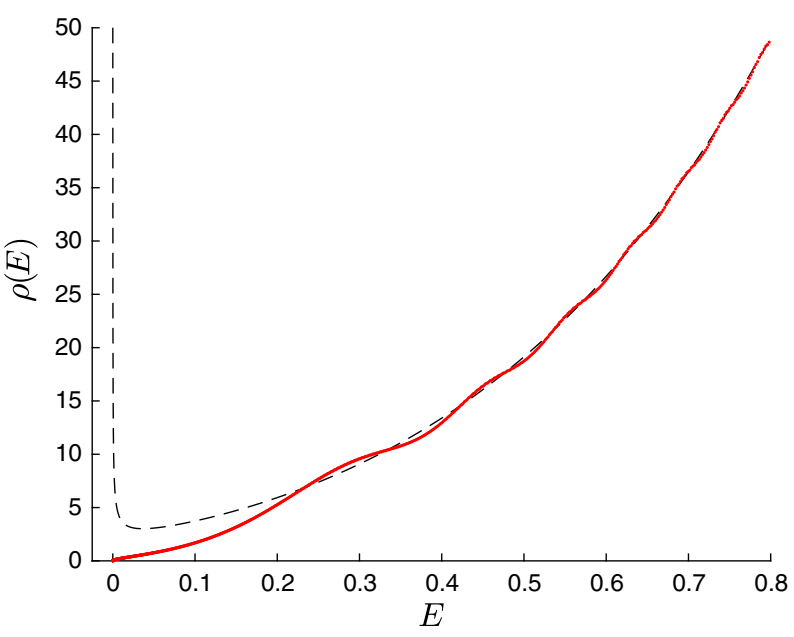

FIG. 2. The full spectral density, computed using the methods of this paper, for the $(2,2)$ model of JT supergravity. The dashed blue line is the disc level result of Eq. (5). Here $\hbar=1$. model in the Altland-Zirnbauer [11] classification scheme, or the case $\Gamma=\frac{1}{2}$ in the notation of Ref. [4]. The result for the companion $(0,2)$ case $\left(\Gamma=-\frac{1}{2}\right)$ will be displayed later (see Fig. 8). As can be seen in Fig. 2, for the $(2,2)$ case, nonperturbative effects entirely erase the characteristic classical peak in the spectrum at low energy, which dramatically alters the "slope" part of the spectral form factor as compared to the analogous result for the $(0,2)$ case, where a peak persists in the full spectrum.

While ordinary JT gravity is important and interesting (and results will be presented for it), a good deal of attention will be given to these two particular models of JT supergravity. They are of particular interest because the nonperturbative physics is more dramatic, in a sense. It was observed in Ref. [6] (and confirmed to be manifest in the minimal model construction of Ref. [4]) that beyond the first one or two leading orders of perturbation theory (depending upon the quantity being computed), the entire topological perturbative series vanishes. Therefore, the nonperturbative effects uncovered in these models (as will be done here) are placed more in stark relief than in other JT gravity systems.

Having shown examples of the key results, the job of the rest of the paper is to explain how to get them, and then to interpret them. The results follow from the nonperturbative construction, proposed in Refs. [3,4], of JT gravity and supergravity in terms of minimal string models (of a special type). The basic idea, building on suggestions in Refs. [5,12], is to reinterpret the JT system as an infinite set of minimal models (nonlinearly) coupled together in a particular way, or equivalently (as explained in Ref. [4]) by turning on an infinite set of operators in the minimal string model obtained by taking the $k \rightarrow \infty$ limit. $^{2}$ Since the full information about the $k$ th minimal string model in question (see Sec. III for a quick review of the essentials) involves solving an order- $2 k+1$ highly nonlinear ordinary differential equation (ODE), this way of defining JT gravity or supergravity involves solving an infinite-order differential equation. This might seem rather daunting, or even formal, but from a pragmatic point of view it is rather straightforward to implement an approximation scheme that allows the computation of an answer to a specific concrete question, to whatever accuracy is desired. The point is that the contribution to the model of successively higher orders of derivatives in the ODE grows smaller with increasing $k$, and so there is a point at which truncating the ODE and solving a finite-order equation will give access to the full spectrum all the way up to a given desired energy, to some required accuracy. In other words, this is hardly any different from computing Feynman diagrams up to some sufficiently high order for some field theory

\footnotetext{
${ }^{2}$ Other recent work exploring connections between the formalism of Liouville theory and minimal strings on the one hand, and JT gravity on the other, includes Refs. [9,13,14].
} 
problem (except that here the formalism is computing nonperturbative physics, and moreover the series is convergent, not asymptotic).

An outline of the paper is as follows: Section II is a brief summary of some of the (now standard) key ideas in the study of JT gravity that will be used in this paper. It is entirely optional for those who know the subject well, but serves to set context, notation, and (perhaps) some motivation. The deconstruction in terms of minimal models will be lightly explained in Sec. III. References [3,4] should be consulted for further details, and the nonperturbative explorations of key toy models presented there. The main task of this paper is to show how to extract nonperturbative results for the full JT (super)gravities. In particular, this section will explain how (using the supergravity examples) the truncation scheme of the previous paragraph works. Section IV will solve the full quantum-mechanical system to yield the nonperturbative spectral density (and hence the partition function) for the supergravity cases. Then, Sec. V turns to the nonperturbative spectral form factor for the supergravities, explaining how it is computed and then displaying several results.

Section VI then discusses the analogous construction and results for a nonperturbative definition of ordinary JT gravity obtained (as first presented in Ref. [3]) by embedding it into a larger framework that it matches perturbatively (at high energy), but which supplies it with nonperturbatively well-behaved low-energy physics.

Since most of the results of this paper come from numerically unpacking the highly nonlinear system of equations (and also using computer algebra to help unpack them), some appendixes are included with some (it is hoped) helpful technical notes and suggestions about the methods employed, for the reader interested in computing these or other results using this formalism. Appendix A presents a numerical study of the spectral form factor of the Airy model (the double-scaled Gaussian Hermitian matrix model) and compares the results to the known exact expressions, showing how the effects of the truncation to a numerical system are extremely well controlled. This serves as a demonstration of the trustworthiness of the numerical results obtained for the JT gravity and supergravity models in the main body of the paper. Section I of Appendix B describes aspects of solving high-order differential equations numerically, and Sec. II of that appendix describes how to solve for the energies and eigenfunctions needed to build the spectrum and spectral form factor. Appendix C lists some important quantities needed in the body of the paper (the Gel'fand-Dikii differential polynomials) and a recursion relation for getting the higherorder expressions.

There are some brief closing remarks in the final section, Sec. VII, with thoughts about the potential application of these methods to other systems.

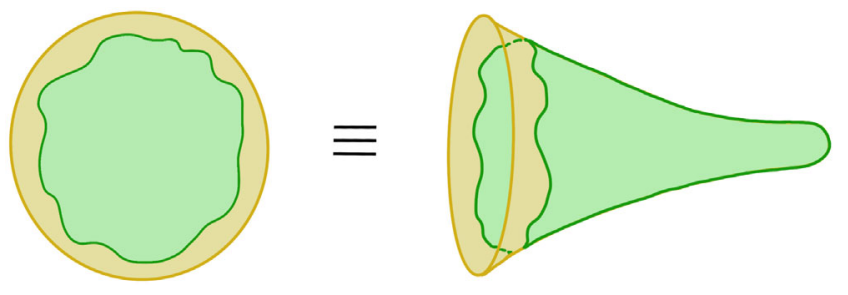

FIG. 3. The "nearly $\mathrm{AdS}_{2}$ " geometry, presented in two equivalent ways.

\section{JT GRAVITY LIGHTNING TOUR}

Although it is a 2D theory of quantum gravity, by virtue of a coupling to a scalar, the dynamics of JT gravity is all on the 1D spacetime boundary. (A good review of much of this is Ref. [15].) The boundary can change its shape while keeping its total length fixed to be the inverse temperature $\beta=1 / T$, the period of Euclidean time. Meanwhile, the bulk spacetime has constant negative curvature (the Ricci scalar $R=-2$ ). So the theory is locally $\mathrm{AdS}_{2}$, and the leading spacetime (disc topology-i.e., no handles or crosscaps, one boundary) is often called "nearly $\mathrm{AdS}_{2}$ " [16-19], in the sense that, e.g., in Poincare coordinates, the boundary is not a fixed circle an infinite distance away, but instead a finite loop of length $\beta$ that is allowed to change its shape. See Fig. 3.

At this order, the dynamics of the loop is controlled by a Schwarzian action [17], and the result is

$$
Z_{0}^{\mathrm{JT}}(\beta)=\frac{e^{S_{0}} e^{\frac{\pi^{2}}{\beta}}}{4 \pi^{1 / 2} \beta^{3 / 2}}=\int_{0}^{\infty} \rho_{0}^{\mathrm{JT}}(E) e^{-\beta E} d E,
$$

related to the disc-order spectral density $\rho_{0}^{\mathrm{JT}}(E)$ by a Laplace transform. There is a JT supergravity generalization of this result $[6,20]$,

$$
Z_{0}^{\mathrm{SJT}}(\beta)=\sqrt{2} \frac{e^{S_{0}} e^{\frac{\pi^{2}}{\beta}}}{\pi^{1 / 2} \beta^{1 / 2}}=\int_{0}^{\infty} \rho_{0}^{\mathrm{SJT}}(E) e^{-\beta E} d E,
$$

defining a disc-order spectral density $\rho_{0}^{\mathrm{SJT}}(E)$. In each case, the densities are given by

$$
\begin{aligned}
& \rho_{0}^{\mathrm{JT}}(E)=e^{S_{0}} \frac{\sinh (2 \pi \sqrt{E})}{4 \pi^{2}}, \quad \text { and } \\
& \rho_{0}^{\mathrm{SJT}}(E)=\sqrt{2} e^{S_{0}} \frac{\cosh (2 \pi \sqrt{E})}{\pi \sqrt{E}}
\end{aligned}
$$

(Henceforth, the redefinition $\sqrt{2} \rho_{0}^{\text {SJT }} \rightarrow \rho_{0}^{\text {SJT }}$ will be made to adapt JT conventions of Ref. [6] to the matrix model normalization to be used here.) The coupling $e^{-S_{0}}$ will be denoted as $\hbar$ in what follows, and indeed it will be the $\hbar$ of a key quantum-mechanical system to appear shortly. One interpretation of $S_{0}$ is that it is simply the 
leading ( $T=0$, disc topology) contribution to the entropy. For the ordinary JT case,

$S=\left(1-\beta \frac{\partial}{\partial \beta}\right) \ln Z_{0}(\beta)=S_{0}+\frac{2 \pi^{2}}{\beta}-\frac{3}{2} \ln \beta+\cdots$.

This leads to a second reason (beyond the one mentioned in the Introduction) to study JT gravity. It is a model of the low-temperature (near-extremal) dynamics of certain higher-dimensional black holes and branes (see, e.g., Refs. [21-25]). For example, the metric of a charged black hole in $d=4$ is well known to become $\operatorname{AdS}_{2} \times S^{2}$ at $T=0$, and the area $A$ of the two-sphere $S^{2}$ sets the $T=0$ entropy: $A=4 S_{0}$. Turning on a small temperature replaces $\mathrm{AdS}_{2}$ with "nearly $\mathrm{AdS}_{2}$," and the horizon area, and hence the entropy, gets corrections. The JT gravity model captures the dynamics of these corrections. (The dynamical scalar represents the deviation of the area away from extremality.) The 2D dynamics can be thought of as containing black holes in its own right as well, worth studying in their own terms. These are, at leading order, the disc geometries already described.

A third reason for studying JT gravity is that it is a lowenergy holographic dual, in a certain sense $[16,18,19,26]$, of a class of 1D quantum systems that exhibit quantum chaos, such as the Sachdev-Ye-Kitaev (SYK) model [17,27,28]. A key diagnostic of the quantum chaotic behavior of the system is the two-point "spectral form factor" $\langle Z(\beta-i t) Z(\beta+i t)\rangle$, which exhibits certain key universal features $[7,8,29,30]$. Starting out at $\left\langle Z(\beta)^{2}\right\rangle$, it decays down a "slope" to a "dip" at during the first epoch of time $t$, and rises along a "ramp" at intermediate times, before leveling off to a "plateau" at late times at a value given by $\langle Z(2 \beta)\rangle$. (See all these features in Fig. 1, but recall that it is not an SYK spectral form factor, but a gravity one; see below.)

The timescales over which these features manifest are important, especially the time to when the plateau sets in, as it gives a measure of how long correlations take to wash away. No single SYK dual cleanly exhibits the universal behavior individually. There are wild oscillations in the spectral form factor at intermediate and late times. ${ }^{3}$ Instead, these features emerge as the time-averaged behavior, as can be seen by averaging over an ensemble of models [31]. An important idea in quantum chaos is the notion that random matrix ensembles should capture the universal features seen in the averaged behavior of a chaotic system (for a review, see Ref. [7]). This led to the suggestion of Refs. [31,32] that a random matrix description of averaged SYK could be available. On the other hand, random matrix models are known to describe, in a "double-scaling" limit [33-36], the sum over surfaces of a $2 \mathrm{D}$ quantum gravity, so this is

\footnotetext{
${ }^{3}$ In the phraseology of the moment, these later eras are "difficult times" for an SYK model.
}
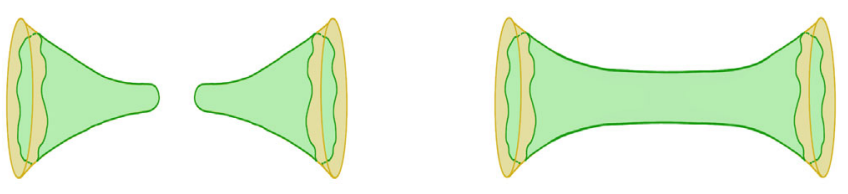

FIG. 4. Black holes vs wormholes.

another way of seeing that there ought to be a dual gravitational description of SYK-like models. This was shown to be more than a coincidence of ideas in Ref. [5], where JT gravity was demonstrated to be explicitly consistent with-order by order in the topological expansion-the properties of a double-scaled matrix model. Reference [6] furnished several more examples and a classification of the possibilities in terms of the ten standard random matrix ensembles.

So, the JT gravity dual (or supergravity dual, for the appropriate generalization of SYK [6,20,37-42]) performs the ensemble average directly. The early-time behavior is controlled by the disconnected diagram constructed of two discs (a pair of $\mathrm{AdS}_{2}$ black holes) plus corrections, while the later ramp and plateau features come from the cylinder diagram (an $\mathrm{AdS}_{2}$ wormhole) [43] plus corrections. See Fig. 4. These amplitudes do not fluctuate chaotically in time, but have smooth behavior to be expected from geometric objects in a theory of gravity. This can be seen already in the leading computation for the cylinder diagram $[5,44]$, which yields a simple $t$ dependence: $\langle Z(\beta-i t) Z(\beta+i t)\rangle \sim \beta^{-1} \sqrt{\beta^{2}+t^{2}}$ gives a rise for the initial part of the ramp behavior in a regime that would already be beset by fluctuations in any given SYK model. For $t \gg \beta$, assuming the transition to the plateau has not yet occurred, this yields a linear rise. In this paper, it will be observed that nonperturbative effects can, depending upon the value of $\hbar$, take over rapidly to generate the ramp, and so the linear part is hardly visible at moderate $\beta$.

As already mentioned, the plateau in the spectral form factor (and the transition to it from the ramp) is a result of perturbative and, especially, nonperturbative corrections to the leading cylinder contribution. The purpose of this paper is to focus on unpacking the nonperturbative definitions of Refs. [3,4] in order to explicitly uncover such effects. Figure 1, already shown above, is a sample of the work reported on in this paper. It is the full spectral form factor for a particular model of JT supergravity. It will be discussed more fully in Sec. V. Now, on to the computations.

\section{CONSTRUCTING JT (SUPER)GRAVITY FROM MINIMAL STRINGS}

The key ingredients are certain double-scaled matrix models that have been used in the past to study certain kinds of "minimal" string theories. (See, e.g., Refs. [44,45] for reviews.) The details of the string theory constructions 
do not matter here. The most important fact to know is that some of the models (a subset of the "one-cut" matrix models) can be described in terms of an associated 1D quantum mechanics problem [46,47], with the Hamiltonian

$$
\mathcal{H}=-\hbar^{2} \frac{\partial^{2}}{\partial x^{2}}+u(x)
$$

where the potential $u(x)$ satisfies a nonlinear ordinary differential equation (ODE) called a "string equation." The key task is to build the correct $u(x)$ for the problem at hand. Once it is known, the full spectral density can be extracted by simply solving the spectrum of $\mathcal{H}$ and evaluating the fully nonperturbative $\rho(E)$, using an expression given in the next section. It is useful to note that in the limit where just the disc-level physics is kept, the spectral density at this order can be written as a simple integral involving the leading part of the potential, $u_{0}(x)=\lim _{\hbar \rightarrow 0} u(x)$ :

$$
\rho_{0}(E)=\frac{1}{\pi \hbar} \int_{0}^{E} \frac{f\left(u_{0}\right)}{\sqrt{E-u_{0}}} d u_{0},
$$

where $f\left(u_{0}\right)=-\partial x / \partial u_{0}(x)$. (This is in a slightly different normalization from that used in Ref. [4].)

Turning back to the ingredients, the minimal models will be labeled by an integer index, $k$. As mentioned before, the models will be combined together to yield the JT (super) gravity. There is a parameter, $t_{k}$, that will be used to turn on the $k$ th model in the mix. The model is turned on if $t_{k}$ is nonzero. The minimal models in question can be obtained ${ }^{4}$ [49-53] by taking the double-scaling limit of models of a complex $N \times N$ matrix $M$, with a potential $V\left(M^{\dagger} M\right)$ (see also footnote 5). The string equation that needs to be solved is

$$
u \mathcal{R}^{2}-\frac{\hbar^{2}}{2} \mathcal{R} \mathcal{R}^{\prime \prime}+\frac{\hbar^{2}}{4}\left(\mathcal{R}^{\prime}\right)^{2}=\hbar^{2} \Gamma^{2},
$$

where the constant $\Gamma$ will be discussed shortly and

$$
\mathcal{R} \equiv \sum_{k=1}^{\infty} t_{k} \tilde{R}_{k}[u]+x
$$

Here, $\tilde{R}_{k}[u]$ is proportional to the $k$ th-order polynomial in $u(x)$ and its $x$ derivatives defined by Gel'fand and Dikii [54]. They have a purely polynomial in $u(x)$ piece, which is $u(x)^{k}$, a purely derivative linear piece, $u(x) x$-differentiated $2 k-2$ times, and then nonlinear mixed terms involving $u(x)$ and its $x$ derivatives. Here, they are normalized so that the coefficient of $u^{k}$ is unity. The first three are

\footnotetext{
${ }^{4}$ These minimal models were later identified by Ref. [48] as the $(4 k, 2)$ superconformal minimal models coupled to gravity with a type-0A projection.
}

$$
\begin{aligned}
& \tilde{R}_{1}[u]=u, \quad \tilde{R}_{2}[u]=u^{2}-\frac{1}{3} u^{\prime \prime}, \quad \text { and } \\
& \tilde{R}_{3}[u]=u^{3}-\frac{1}{2}\left(u^{\prime}\right)^{2}-u u^{\prime \prime}+\frac{1}{10} u^{\prime \prime \prime \prime},
\end{aligned}
$$

where a prime denotes an $x$ derivative times a factor of $\hbar$. It will transpire that $\tilde{R}_{4}, \tilde{R}_{5}, \tilde{R}_{6}$, and $\tilde{R}_{7}$ will be used in this paper too, but since they are rather lengthy, some are listed in Appendix C, along with methods for generating others if needed.

The boundary condition that ensures good nonperturbative behavior is, for each model,

$$
\begin{aligned}
& u(x) \rightarrow 0 \quad \text { as } x \rightarrow+\infty, \\
& u(x) \rightarrow(-x)^{\frac{1}{k}} \text { as } x \rightarrow-\infty .
\end{aligned}
$$

Note the presence of $\hbar=e^{-S_{0}}$ in the string equation (and the various quantities that make it up). It is very useful for separating the classical parts from the rest, by sending $\hbar \rightarrow 0$, or equivalently, dropping derivatives. For the study of nonperturbative physics, solutions $u(x)$ of the equation will be extracted for $\hbar=1$. Several results presented in figures to come will be for this value, because it allows the nonperturbative effects to be writ large in the results (for spectra, etc.), and therefore seen easily. When it is instructive to do so, comparison to results with $\hbar$ dialed down will be discussed. It is interesting that it is in fact more difficult to solve the string equations for smaller $\hbar$. This is because when derivatives have smaller coefficients, they are allowed to fluctuate more, contributing to the sensitivity when solving these highly nonlinear equations, as will be discussed later. (This increased difficulty to get smaller $\hbar$ results has the character of a sort of strong/weak coupling duality, in fact.)

Turn now to the constant $\Gamma$ in the string equation (9). With it present, the matrix model is in the $(2 \Gamma+1,2)$ class in the $(\boldsymbol{\alpha}, \boldsymbol{\beta})$ Altland-Zirnbauer classification of matrix ensembles. ${ }^{5}$ The two choices $\Gamma= \pm \frac{1}{2}$ will mostly be

\footnotetext{
${ }^{5}$ Double scaling means that in the matrix model [49-53] of the complex matrix $M$, the size $N$ is taken to infinity while couplings in the potential $V\left(M^{\dagger} M\right)$ are tuned to certain critical [55] values. Diagonalizing $M$ turns this into a problem involving its eigenvalues $\lambda_{i}(i=1 \ldots N)$ at a cost of introducing a van der Monde determinant $J=\prod_{i<j}\left(\lambda_{i}^{2}-\lambda_{j}^{2}\right)^{2}$ for the Jacobian. The effective Dyson gas problem for the $\lambda_{i}^{2}$ can be thought of as existing on the positive real line, with a wall at zero. The constant $\Gamma$ in Eq. (9) can be thought of as arising from the coefficient of a logarithmic term in the potential of the model (see, e.g., Ref. [56]), and as such, results in an extra factor $\lambda_{i}^{2 \Gamma}$ in the effective integration measure over the $i$ th eigenvalue, giving $\prod_{i} \lambda_{i}^{2 \Gamma+1} d \lambda_{i}$. With the factor $J$ included, the model is seen to be in the $(2 \Gamma+1,2)$ class in the $(\boldsymbol{\alpha}, \boldsymbol{\beta})$ Altland-Zirnbauer classification of matrix ensembles, defined for $\boldsymbol{\alpha}=0,1,2$. Actually, $\Gamma$ can be more general integers or half-integers than just these values.
} 
considered in this paper, and the two JT supergravity models discussed here will be labeled $(2,2)$ and $(0,2)$.

A particular (say, the $m$ th) minimal model can be studied by setting all the $t_{k}$ 's to zero except for $k=m$. As discussed in the previous two papers $[3,4]$, the $m=1$ case in particular is important, as it models the low-energy tail of the eigenvalue distribution very well. To get the full behavior, all of the $t_{k}$ 's must be turned on in a particular combination. For example, in the case of JT supergravity, the combination (derived in Ref. [4]) is

$$
t_{k}=\frac{\pi^{2 k}}{(k !)^{2}} \text {. }
$$

So, all the infinite models are turned on, and the string equation becomes a highly complicated object. But the purpose of this paper is to show that physics can be readily extracted nonetheless.

Here is the reason why. First, look at the disc level. The string equation is expression (9) with the three parts with $\hbar^{2}$ in front of them removed, and the solution for $u_{0}(x)$ comes in two branches. Either $u_{0}(x)=0$, or

$$
\mathcal{R}=\sum_{k=1}^{\infty} t_{k} u_{0}^{k}+x=0,
$$

corresponding to the asymptotics given in Eq. (12). This equation has $u_{0}(x)=0$ at $x=0$, and so the two branches join at $x=0$. For the second branch, the $x<0$ regime, the combination [Eq. (13)] of $t_{k}$ 's amounts to $f\left(u_{0}\right)=$ $\pi I_{1}\left(2 \pi \sqrt{u_{0}}\right) / \sqrt{u_{0}}$ in Eq. (8), yielding the part of the spectral density expanded in positive powers of $E$. The simple $E^{-\frac{1}{2}}$ part comes from the $u_{0}=0$ behavior. Integrating $f\left(u_{0}\right)$ with respect to $u_{0}$, or simply by looking at Eq. (14), the explicit potential that gives JT supergravity on the disc is given by the equation

$$
x=1-I_{0}\left(2 \pi \sqrt{u_{0}}\right),
$$

where $I_{0}(s)$ is the zeroth modified Bessel function of $s$. This is a remarkably simple form.

The issue of tractability becomes the simple issue of how well this potential can be approximated by truncating to a finite number of $t_{k}$ 's. The answer boils down to what maximum energy scale $E$ one wants to know the spectrum up to, and to what accuracy. As an example, the full classical potential [Eq. (15)] is plotted in Fig. 5 alongside two truncations.

The first truncation contains just $t_{1}$ and $t_{2}$ :

$$
x=-\pi^{2} u_{0}-\frac{1}{4} \pi^{4} u_{0}^{2},
$$

and it is clear that it is a good approximation for energies up to approximately $E \sim 0.1$, after which it begins to deviate considerably. The next example truncation adds $t_{3}$ and $t_{4}$ :

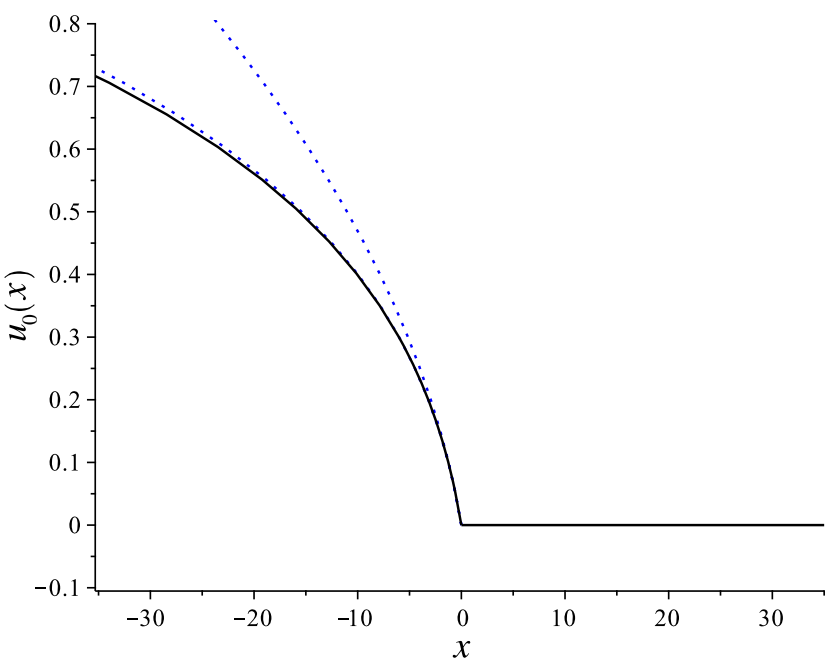

FIG. 5. The complete classical potential for JT supergravity (solid line). The uppermost dotted line is a truncation up to $t_{2}$, while the lower dotted line is a truncation up to $t_{4}$.

$$
x=-\pi^{2} u_{0}-\frac{1}{4} \pi^{4} u_{0}^{2}-\frac{1}{36} \pi^{6} u_{0}^{3}-\frac{1}{576} \pi^{8} u_{0}^{4}
$$

and for energies up to order $E \sim 0.5$ it serves rather well. Further improvements come by adding higher orders.

The next issue to appreciate is how much the solution changes when all the nonperturbative corrections are included. For all $k$, a shallow well can develop in the central region (slightly to the right of $x=0$ ). Crucially, moving away from that region, the deviation of the solution from the disc-level behavior rapidly dwindles, as it matches on to the asymptotic behavior. The same is true for the coupled solution. Moreover, as can be seen from Eq. (13), the form of the $t_{k}$ as $k$ grows is such that good approximations at the disc level can be found by adding only a small number of minimal models, for a given needed accuracy. For larger $E$ 's, the solution becomes hard to distinguish from the classical result, and in that case the exact classical potential can be used, to a good approximation.

The highest truncation levels chosen for the purposes of this paper were to keep all the minimal models up to $k=6$ (this section) and $k=7$ (for Sec. VI), although very good results were obtained for lower-order truncations too. Since $R_{7}[k]$ has the 12th-order derivative of $u(x)$ in it, the string equation (9) is a 14th-order differential equation. (It is 12 th-order for the $k=6$ truncation.) In general, it is easier to take a derivative of the string equation, whereupon an overall factor of $\mathcal{R}$ can be divided out, reducing some of the nonlinearity somewhat, at the expense of an increase in the order. For the boundary conditions in question, a 15th- (or 13th-) order differential equation is not too hard to solve numerically, with care. Some suggestions and notes are given in Sec. I of Appendix B, for those who wish to carry out their own computations using this framework. 


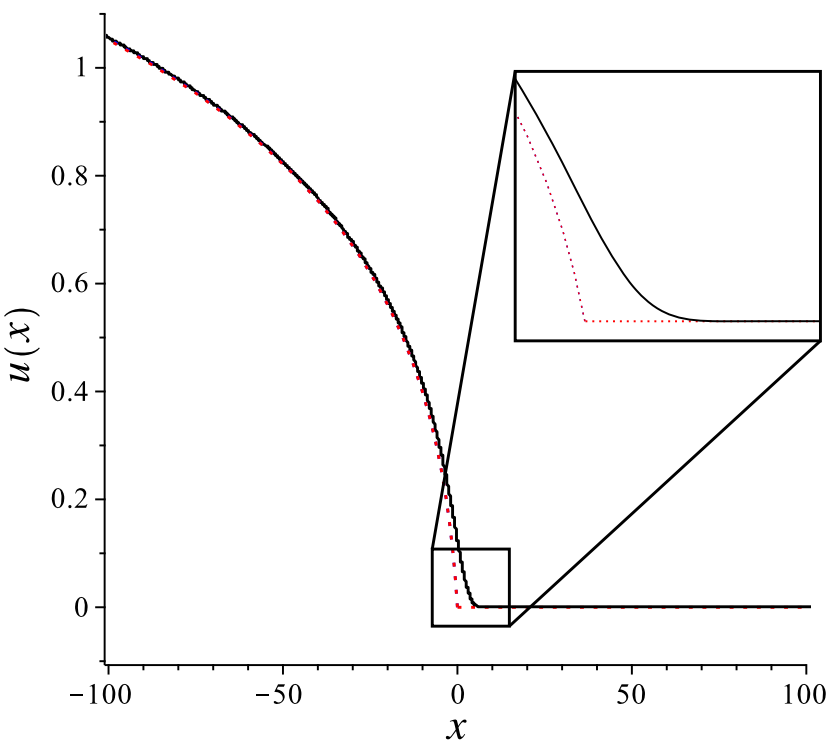

FIG. 6. The $\Gamma=+\frac{1}{2}$ solution (solid line) of the string equation for truncation up to $t_{6}$. The inset shows a closeup of the smooth transitional region in the interior. For comparison, the full classical solution line is shown too (dotted line).

Part of the full nonperturbative potential $u(x)$ for the truncation to $k=6$ is displayed in Fig. 6 .

This is for the case $\Gamma=+\frac{1}{2}$ i.e., the $(2,2)$ JT supergravity. (It was solved between $x=-200$ and $x=+200$.) Notice that it approaches the classical solution and agrees with it rather well up to (and beyond, it turns out) $E \sim 1.3$, and so the full solution, out to beyond the $x \simeq-100$ shown, can be used to capture the spectrum of $(2,2)$ JT supergravity

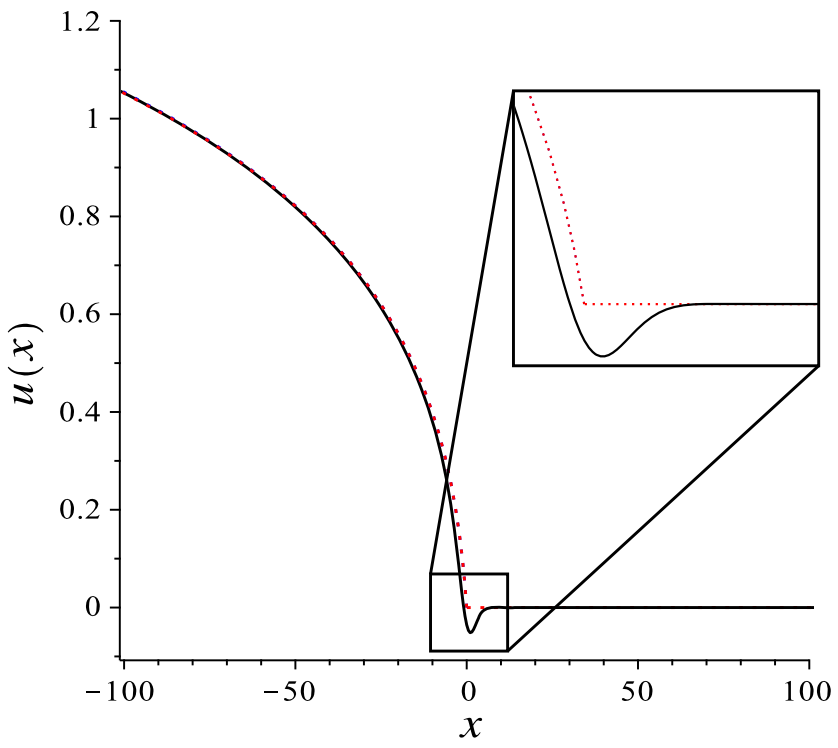

FIG. 7. The $\Gamma=-\frac{1}{2}$ solution (solid line) of the string equation for truncation up to $t_{6}$. The inset shows the smooth well that developed in the interior. For comparison, the full classical solution is shown too (dotted line). with good accuracy (see more in Sec. II of Appendix B on how to do this). It is (relatively) easy to do better, if desired, but little visible change was noticed in going to higher orders, in exchange for accessing only a slightly larger maximum energy.

Figure 7 shows the solution for $\Gamma=-\frac{1}{2}$, which will be used to study the properties of the $(0,2)$ JT supergravity.

This solution has a well in the interior (as is quite typical of these solutions), and so slightly more rapid changes take place there. Rather than use numerical methods to solve for this directly (which are inevitably more sensitive to error in this case), a handy solution-generating technique derived in Ref. [57] was used, ${ }^{6}$ that allowed it to be generated from the $\Gamma=+\frac{1}{2}$ solution already found. See Sec. I of Appendix B for more on this.

\section{THE SPECTRAL DENSITY}

\section{A. General remarks}

The next step is to solve the full spectral problem for the Hamiltonian $\mathcal{H}$, given the potential $u(x)$ found in the previous section by solving the (truncated) string equation. The relation between the spectrum of $\mathcal{H}$ and the JT partition function is as follows: From the minimal string perspective, the JT (super)gravity partition function is simply $[5,12]$ the expectation value of a "macroscopic loop" of length $\beta$. The technology for working this out was derived long ago in Ref. [46]. (Reference [44] unpacks the formalism in a useful review.) It is the trace of the exponentiated $\mathcal{H}$, with a projection $\mathcal{P}$ inserted:

$$
Z(\beta)=\operatorname{Tr}\left(e^{-\beta \mathcal{H}} \mathcal{P}\right)
$$

where the operator $\mathcal{P} \equiv \int_{-\infty}^{\mu} d x|x\rangle\langle x|$, and the upper limit $\mu$ will be discussed shortly. Inserting a complete set of states

$$
\int d \psi|\psi\rangle\langle\psi|=1
$$

yields

$$
\begin{aligned}
Z(\beta) & =\int_{-\infty}^{\mu} d x\left\langle x\left|e^{-\beta \mathcal{H}}\right| x\right\rangle \\
& =\int_{-\infty}^{\mu} d x \int d \psi\left\langle x\left|e^{-\beta \mathcal{H}}\right| \psi\right\rangle\langle\psi \mid x\rangle \\
& =\int_{-\infty}^{\mu} d x \int d \psi_{E}\left\langle x \mid \psi_{E}\right\rangle\left\langle\psi_{E} \mid x\right\rangle e^{-\beta E} \\
& =\int d E e^{-\beta E} \rho(E),
\end{aligned}
$$

\footnotetext{
${ }^{6}$ It is inherited from the rather rich underlying Kortewegde Vries $(\mathrm{KdV})$ hierarchy integrable structure that underpins this entire formalism.
} 
where

$$
\rho(E)=\int_{-\infty}^{\mu} \psi(x, E) \psi^{*}(x, E) d x
$$

is the spectral density.

To understand the upper limit $\mu$, return to the leadingorder expression for the density given in Eq. (8), and change variables to $x$. The solid black line in Fig. 5 is a reminder of the behavior of $u_{0}(x)$. The part of the integral where $u_{0}(x)=0$ begins at $x=0$ and extends to positive $x$ to some value denoted $\mu$, while (at a given $E$ ) the lowest value for $x$ is set by the turning point where $E=u(x)$, at a position denoted $-\left|x_{0}\right|$, and so

$$
\rho_{0}(E)=\frac{1}{\pi \hbar} \int_{-\left|x_{0}\right|}^{\mu} \frac{d x}{\sqrt{E-u_{0}(x)}}
$$

In the full quantum expression [Eq. (21)], there are contributions to the spectral density from the whole integration range down to $x=-\infty$. Intuitively, this is because wave functions penetrate to the left beyond the classical turning point $E=u(x)$. [Often, in the classical expression (22), the lower limit is also written as $x=-\infty$ with the understanding that only the real part of the integral contributes to $\rho_{0}$. $]^{7}$ The role of $\mu$ becomes clear from focusing on just the contribution from the $u_{0}(x)=0$ part in Eq. (22). It adds $\mu /(\pi \hbar \sqrt{E})$ to the classical spectral density. Physically, $\mu$ controls how much the classical spectral density is "piled up" against the natural wall at $E=0$ that stops the energies in the complex matrix models from going negative (see footnote 5). Vanishing $\mu$ corresponds to just touching the wall. In the normalization of Eq. (5) (without the $\sqrt{2}$ ), the value of $\mu$ is 1 , and this will be used for much of the rest of this paper. (Reference [4] uses the value $\mu=2$.) However, it is useful for later to write a more general classical spectral density with a different $\mu$ :

$$
\rho_{0}^{\mathrm{SJT}}(E, \mu)=\frac{1}{\pi \hbar}\left[\frac{\cosh (2 \pi \sqrt{E})}{\sqrt{E}}+\frac{\mu-1}{\sqrt{E}}\right] .
$$

\footnotetext{
${ }^{7}$ Of course, the integrand of Eq. (21) is not written in terms of $E$ and the full $u(x)$, but in terms of the wave functions. The precise connection between the two expressions is via the diagonal of the resolvent, $(\mathcal{H}-E)^{-1}$, of $\mathcal{H}$, denoted $\hat{R}(x)$. It can be built out of $\psi(x, E) \psi^{*}(x, E)$, using standard Green function methods. Gel'fand and Dikii [54] derived an equation for $\hat{R}(x)$ which is $4(u(x)-E) \hat{R}^{2}-2 \hat{R} \hat{R}^{\prime \prime}+\left(\hat{R}^{\prime}\right)^{2}=1$. The leading-order solution comes again from dropping derivatives, and is $\hat{R}(x)= \pm(2 \sqrt{u(x)-E})^{-1}$. In the normalization of this paper, $\rho_{0}=(2 / \pi \hbar) \operatorname{Im} \int \hat{R}(x) d x$, yielding the form in Eq. (22).
}

\section{B. Computation}

Now to the matter of computing the full spectral density [Eq. (21)]. Just as in Refs. [3,4], a matrix Numerov method [58] was used to solve for the spectrum of $\mathcal{H}$. Conceptually, it is a simple problem of finding eigenvalues and eigenfunctions for a Schrödinger operator in 1D. The wave functions are free and oscillatory to the far right $(x>0)$; begin to feel the presence of the potential as they move further into the interior (it starts as a $1 / x^{2}$ dependence); and then once they hit the potential, there is an exponential decay to the left $(x<0)$. As a guide to extracting them with good accuracy, some extra suggestions and notes for interested readers are given in Sec. II of Appendix B. The same normalization method as the one used in Ref. [3] was used for the resulting eigenfunctions. The key point explained there is that in the far $x>0$ region, wave functions are known to asymptote to a simple form involving the Bessel function of order $\Gamma$, where the normalization can be analytically chosen to yield the correct contribution to the disc-level spectral density.

The outcome of the numerical spectrum solving was approximately 1000 accurate normalized wave functions and their energies, for use in constructing the density in this section, and the spectral form factor in the next. Using them, the spectral density can be constructed using a simple trapezoidal integration to implement Eq. (21), and the result (for $\mu=1$ ) is shown in Fig. 2 for $\Gamma=\frac{1}{2}$ and in Fig. 8 for $\Gamma=-\frac{1}{2}$. Plotted alongside the (dense) line of dots (the computed spectral data) is a dashed line showing the disclevel spectral density. Strikingly, the $1 / \sqrt{E}$ divergence present at disc level is erased entirely by nonperturbative effects in the $\Gamma=\frac{1}{2}(2,2)$ case, but not in the $\Gamma=-\frac{1}{2}(0,2)$

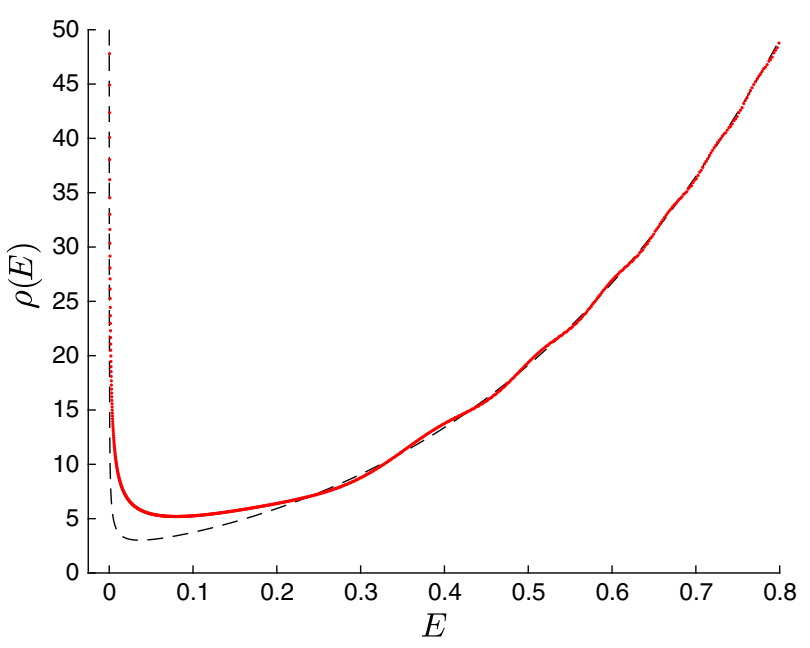

FIG. 8. The full spectral density (made out of red dots), computed using the methods of this paper, for the $(0,2)$ model of JT supergravity. The dashed blue line is the disc-level result of Eq. (5). Here, $\hbar=1$. The companion result for the $(2,2)$ model is in Fig. 2. 
case. As discussed in Refs. [4,6], for $\Gamma= \pm \frac{1}{2}$, there are no perturbative corrections to the spectral density beyond the disc, so all the differences here are due to nonperturbative physics, which makes these JT supergravity cases particularly interesting to study when investigating the results of nonperturbative physics. This stark difference will be reflected in comparisons of the spectral form factor, to be studied in the next section.

Satisfyingly, what have appeared here for the full $(2,2)$ and $(0,2)$ cases are actually grown-up versions of what was discussed for two baby (Bessel) models in Ref. [6], and so this constitutes a nice consistency check of the methods of this paper. The Bessel special cases are models of the very low-energy tip of the spectral density - the part that (classically) pushes up against the $E=0$ wall, as mentioned earlier. Generalizing to include arbitrary positive $\mu$ (see also Refs. [3,4]), the full nonperturbative Bessel spectral density is (after a change of conventions from those papers)

$$
\rho(E)=\frac{\mu}{\pi \hbar \sqrt{E}}+\frac{\sin \left(-\frac{\pi}{2} \alpha+\frac{2 \mu \sqrt{E}}{\hbar}\right)}{2 \pi E},
$$

where (as a reminder) $\alpha=2 \Gamma+1$ takes the values 0 and 2. The leading part is the disc contribution, and all other perturbative contributions vanish. The oscillating term is the full nonperturbative contribution. At $E=0$ for $\alpha=2\left(\Gamma=\frac{1}{2}\right)$, there is the aforementioned cancellation between the divergent disc contribution and the $E \rightarrow 0$ piece of the oscillating nonperturbative part. This is what is now seen to be present (as it ought to be) in the full JT supergravity spectral density computed explicitly above.

\section{A special formula, and a tale of instantons}

Actually, it is possible to go considerably further than the Bessel tail comparison. The structure of the Bessel models above (along with various other technical features of the matrix model recursion relations) led Stanford and Witten to suggest (see Appendix E of Ref. [6]) that more generally, the JT supergravity spectral density for the $(0,2)$ and $(2,2)$ cases should closely approximate the form ${ }^{8}$

$$
\rho(E) \approx \rho_{0}(E)+\frac{\sin \left(-\frac{\pi}{2} \alpha+\pi \int_{0}^{E} \rho_{0}\left(E^{\prime}\right) d E^{\prime}\right)}{2 \pi E} .
$$

(Note that their conventions were adapted to match those of the current paper.) Again, the perturbative terms beyond the disc vanish. The argument of the sinusoidal nonperturbative piece is determined in terms of the disc

\footnotetext{
${ }^{8}$ The author thanks Douglas Stanford for asking a helpful question about this issue after the first version of this manuscript appeared.
}

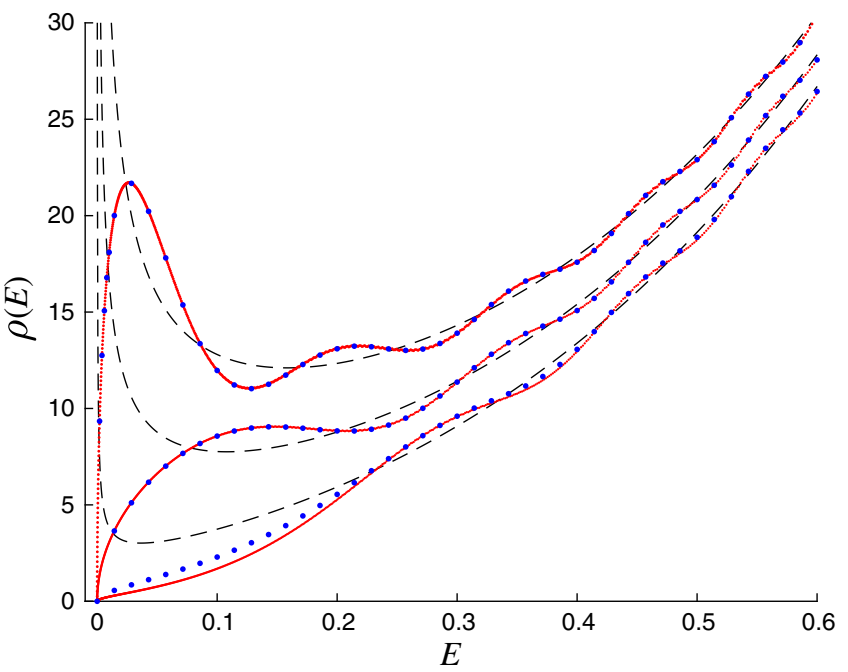

FIG. 9. Comparison of the full spectral density for the $(2,2)$ model of JT supergravity with Eq. (25) for the leading form (dark blue dots). The dashed line is the disc-level result [Eq. (23)]. The cases $\mu=1,5$, and 10 are shown (lower to upper). Here, $\hbar=1$.

contribution. ${ }^{9}$ Crucially, they note that this does not include possible instanton corrections, nonperturbative physics of a form not accounted for by the sinusoidal modulation (hence the use of the $\approx$ sign in the above).

While the expression above was written for the specific value $\mu=1$ ( $\mu$ is not a variable parameter in Ref. [6]), other values of $\mu$ [see below Eq. (21) for its explanation] are quite readily incorporated by this form. This is already obvious for the simple Bessel prototype [Eq. (24)], but further evidence comes from inserting into it the expression (23) for $\rho_{0}(E, \mu)$ written earlier for the disc-level JT supergravity density for other $\mu$ values. Plotting this analytical result against the numerical results for the spectral density obtained using this paper's methods yields a remarkable agreement, as shown in Figs. 9 and 10 for $\Gamma=\frac{1}{2}$ and $\Gamma=-\frac{1}{2}$, for the values $\mu=1,5$, and 10 . As a reminder, the red lines are each made of $\sim 1800$ points individually computed using the truncation and numerical methods outlined, while the blue dots are samples of the analytical formula [Eq. (25)]. It is very striking how well they agree.

The areas of disagreement suggest interesting physics, in fact. For smaller $\mu$ (e.g., see the case of $\mu=1$ in each figure), it is easy to see disagreement. It is especially quite visible at smaller energies (although at $E=0$ they agree, as they must, since this is covered by the Bessel cases above). The clear pattern in the discrepancy is suggestive of physics and not numerical inaccuracies.

For example, the fact that the disagreement rapidly disappears as $\mu$ increases is striking. Nothing about the

\footnotetext{
${ }^{9}$ In fact, it is easy to guess generalizations of this formula for other half-integer $\Gamma$ 's, which seem to give sensible physics using the present methods. It would be interesting to test them.
} 


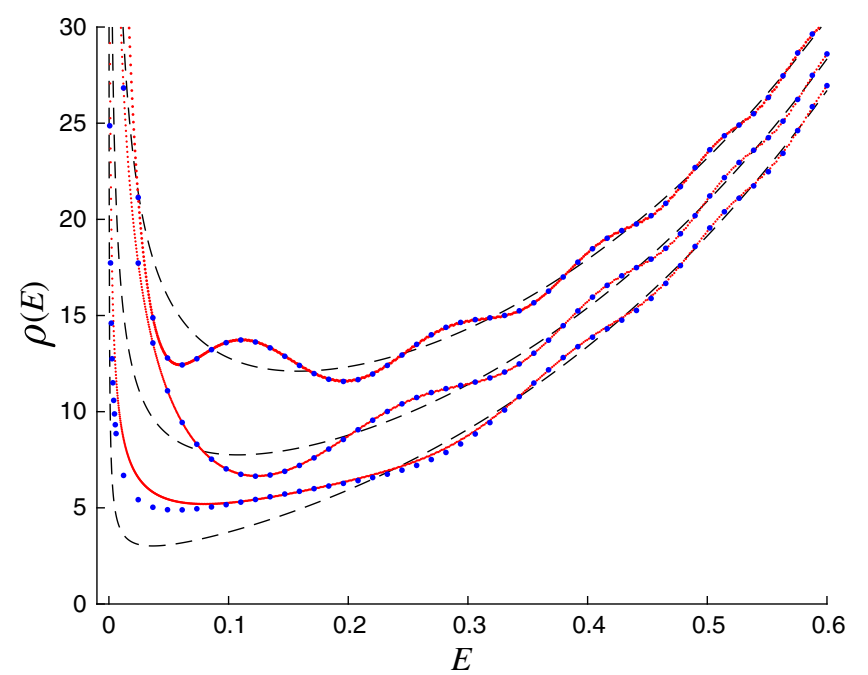

FIG. 10. Comparison of the full spectral density for the $(0,2)$ model of JT supergravity with Eq. (25) for the leading form (dark blue dots). The dashed line is the disc-level result [Eq. (23)]. The cases $\mu=1,5$, and 10 are shown (lower to upper). Here, $\hbar=1$.

numerical methods should show this sort of systematic dependence. As a further example, the form of the disagreement suggests that there is a change in the amplitude of the sinusoidal modulation, again a pattern that is hard to reproduce with mere numerical systematics. A natural guess is that there are further multiplicative factors in the nonperturbative parts that are not present in the analytic formula. They must be small, and of an instanton form (as already anticipated in remarks in Ref. [6]).

In the $k$ th minimal model, the height of the effective potential barrier for one eigenvalue in the background of all the others yields the action of the instanton effects [59] (they are "ZZ-branes" in the modern parlance [60]). Alternatively, it can be computed using a WKB analysis of the string equation itself. This was all worked out long ago for the complex matrix models in Ref. [52] (see Ref. [61] for a review and more contemporary presentation), and the dependence of the action on $\mu$ and $\hbar$ is $\left(\frac{4 k}{2 k+1}\right) \mu^{1+\frac{1}{2 k}} / \hbar$. Therefore, a dependence $\exp (-2 \mu / \hbar)$ should be expected for instanton corrections to JT supergravity constructed out of minimal models in the manner of this paper.

To test this, a rough estimate of the dependence was made by measuring the size of the deviations of the type seen in Figs. 9 and 10 for some sample cases, as $\mu$ is varied from 0 to 5. The deviations fall rapidly in that range (as is evident from the figures), and a logarithmic plot of them against $\mu$ yields a straight line of negative unit slope to good (better than 1\%) accuracy. While this can be made more precise, it is already a strong indication that the instanton effects of the expected form are present and accounted for. It will be interesting to explore this further, but this will be left for later work.
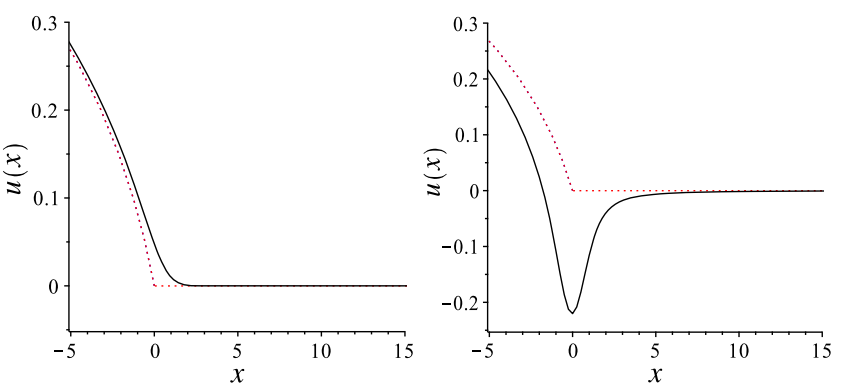

FIG. 11. The solid lines show close-ups of the $\Gamma=\frac{1}{2}$ or $(2,2)$ solution (left) and the $\Gamma=-\frac{1}{2}$ or $(0,2)$ solution (right) of the string equation for truncation up to $t_{6}$, for $\hbar=\frac{1}{5}$. For comparison, the full classical solution is shown too (dotted lines).

\section{Going to weak coupling}

Before moving on, it is worth showing the effect of going to weak coupling by reducing $\hbar$. The spectra shown so far are for $\hbar=1$, and working in this regime has the advantage of making much of the nonperturbative physics readily visible. Nevertheless, it is useful to be able to gain access to weaker coupling, not the least because certain dual systems of interest [such as SYK-type models, or large black holes (where $S_{0}$ is large)] may be in that regime.

It is possible to reduce the value of $\hbar$, but it is at the expense of not being able to access as high energies in the spectrum for a given truncation, unless there is a compensation in terms of increased computational effort (working on a finer grid and producing more wave functions). Additionally, solving the string equation takes more care, as already mentioned, because the derivatives are more in play. Nevertheless, progress is possible. For example, dialing down to, e.g., $\hbar=\frac{1}{5}$, the string equations can be coaxed to produce the solutions shown (just as close-ups, for brevity) in Fig. 11. These should be compared to what was shown in the insets of Figs. 6 and 7. For the $(2,2)$ case, the curve turns the corner more sharply (showing the increased role of the derivatives), while for the $(0,2)$ case, the well is far deeper and narrower (these two features come together to continue to ensure there are no bound states).

It is easy to imagine how these developments continue in order to get to the classical limit $\hbar \rightarrow 0$ (the red dotted line in the figures). The $(2,2)$ curve turns the corner ever more sharply, and for the $(0,2)$ curve, the well deepens and narrows and eventually is entirely squeezed away.

The techniques already described in the previous two sections can be carried out to study the spectral density, and the result in Fig. 12 displays the results (red dots) for the $\Gamma=\frac{1}{2}$ or $(2,2)$ case, for the cases $\mu=1$ and 5 . (The $\mu=10$ case, which rises to 508 vertically, was omitted for clarity.) Again, the blue dots are samples of the analytic formula (25) with Eq. (23) input for $\rho_{0}(E, \mu)$ (now $\hbar=\frac{1}{5}$ is inserted), showing again how well this procedure works. Notice that, in accordance with the fact that the coupling is weaker, the 


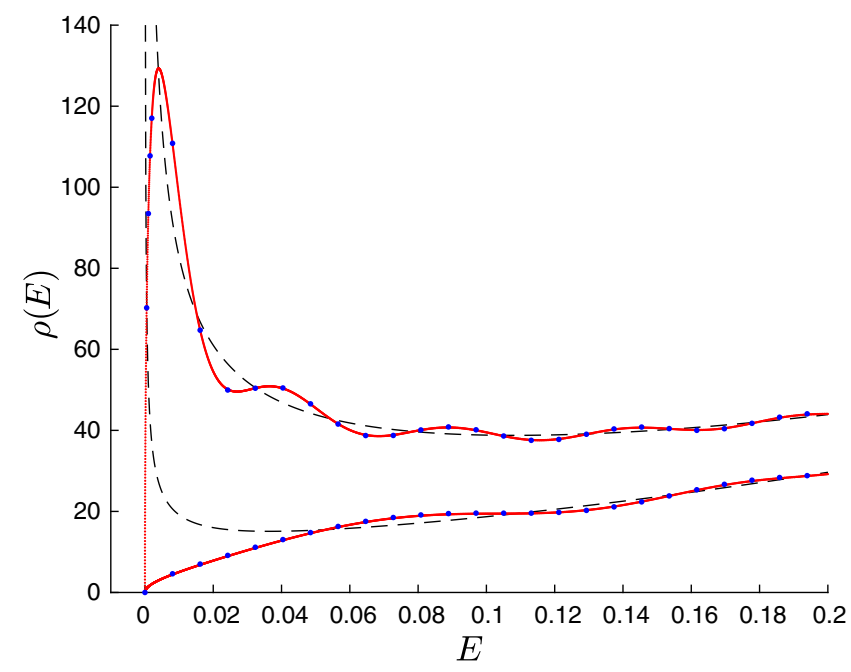

FIG. 12. Two examples ( $\mu=1$, lower; $\mu=5$, upper) of the full spectral density for the $(2,2)$ model of JT supergravity, for $\hbar$ reduced to $\frac{1}{5}$. The (blue) dots are points from Eq. (25). In each case, the dashed line is the disc result of Eq. (23). Cf. the $\hbar=1$ cases of Fig. 9.

$\exp (-\mu / \hbar)$ instanton effects described in the previous section, which would appear as deviations of the red curves from the blue dots, are too small to seen here.

Comparing to the $\hbar=1$ curves in Fig. 9, the quantum undulations are smaller, for generic $E$, and the curves stray less from the classical curve, beginning marked deviations only at lower energies compared to the $\hbar=1$ situation, until the curve falls quickly to zero. This is as it should be.

\section{THE SPECTRAL FORM FACTOR}

\section{A. General remarks}

As mentioned in the brief review of Sec. II, the spectral form factor is derived from the two-point function of $Z(\beta)$. This has two parts, a disconnected piece $\langle Z(\beta)\rangle\left\langle Z\left(\beta^{\prime}\right)\right\rangle$ and a connected piece $\left\langle Z(\beta) Z\left(\beta^{\prime}\right)\right\rangle$. In the old matrix model language, the connected piece is the connected correlator of two "macroscopic loops," and this is readily written down as $[44,46]$

$$
\begin{aligned}
\left\langle Z(\beta) Z\left(\beta^{\prime}\right)\right\rangle & =\operatorname{Tr}\left(e^{-\beta \mathcal{H}}(1-\mathcal{P}) e^{-\beta^{\prime} \mathcal{H}} \mathcal{P}\right) \\
& =\operatorname{Tr}\left(e^{-\left(\beta+\beta^{\prime}\right) \mathcal{H}}\right)-\operatorname{Tr}\left(e^{-\beta \mathcal{H}} \mathcal{P} e^{-\beta^{\prime} \mathcal{H}} \mathcal{P}\right) \\
& =Z\left(\beta+\beta^{\prime}\right)-\int d E \int d E^{\prime} \rho\left(E, E^{\prime}\right) \rho^{*}\left(E^{\prime}, E\right),
\end{aligned}
$$

where $\mathcal{P}$ is discussed below Eq. (18) and

$$
\rho\left(E, E^{\prime}\right)=\int_{-\infty}^{\mu} d x \psi^{*}(x, E) \psi\left(x, E^{\prime}\right) .
$$

The quantity $\mu$, discussed in the previous section, will be set to unity henceforth, corresponding to having the disc-level spectral density given in Eq. (5) (without the overall $\sqrt{2}$ ).

\section{B. A phase transition}

Set $\beta=\beta^{\prime}$ for a while. Generically, the disconnected part (corresponding to two black holes) and the connected part (a wormhole) are worth studying in their own right as distinct sectors of the quantum gravity that compete for dominance [62] as a function of $\beta$. The disconnected part, being the square of the partition function, rapidly decreases with increasing $\beta$, while the connected part increases. At some point $\beta_{\mathrm{cr}}$, there is a transition, and the connected diagram becomes more dominant. This is also true in the complete (not just perturbative) theory discussed here. This is all nicely under control in the current definitions of JT supergravity. The spectrum has been computed in the previous section, and so all the elements in Eq. (26) are readily computable to the desired accuracy. Figure 13 shows a plot of the ( $\log _{10}$ of the) connected and disconnected pieces-with all perturbative and nonperturbative contributions included-as a function of $\beta$, for the $(2,2) \mathrm{JT}$ supergravity case, showing the transition at $\beta_{\mathrm{cr}}=13.56$. A similar computation (yielding a similar graph, omitted) shows that $\beta_{\mathrm{cr}}=28.30$ for the $(0,2)$ JT supergravity case.

Notably, for the $(0,2)$ case, the amplitudes are over an order of magnitude larger. This is a striking effect attributable entirely to nonperturbative effects. The $(2,2)$ JT supergravity has, as mentioned in the previous Sec. IV, nonperturbative effects that cancel the $1 / \sqrt{E}$ behavior at low energy, coming from the leading disc amplitude. The $(0,2)$ version does not cancel this away, and so while for larger $E$ the two models' spectra are roughly similar

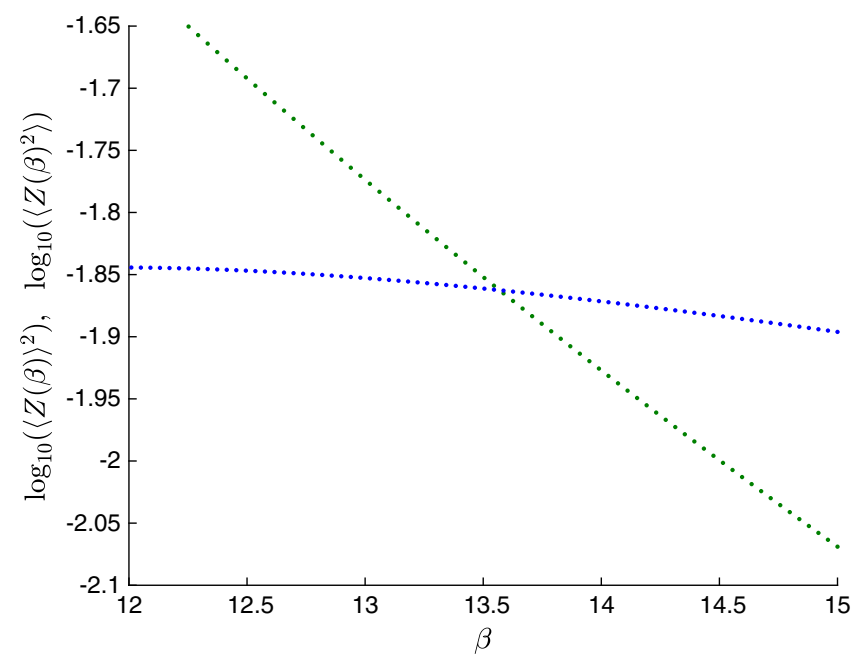

FIG. 13. The disconnected (starting higher at the left) vs the connected (lower) two-point function of the partition function as a function of $\beta$ at $\hbar=1$, showing a phase transition at $\beta_{\mathrm{cr}} \simeq 13.56$. 
(see Figs. 2 and 8), there is an enhancement at low $E$ that means much larger contributions to the partition function for any fixed $\beta$. This marked difference between the nonperturbative physics will make a major appearance in the temporal behavior of the spectral form factor too, studied next.

\section{Time dependence}

The above computation serves as a useful guide for what to expect for the spectral form factor, which tracks the correlation function over time. This is done by setting $\beta \rightarrow \beta+i t$ and $\beta^{\prime} \rightarrow \beta-i$, with $\beta$ fixed, studying the dependence on $t$. The fixed $\beta$ can be above or below $\beta_{\mathrm{cr}}$. The disconnected part will start out as the squared partition function and then decrease with $t$. This is the "slope" behavior of the spectral form factor. On the other hand, looking at Eq. (26), it can be seen that the connected part has a $t$-independent part, $Z(2 \beta)$, from which is subtracted a positive piece which gets small at large $t$, with significant contributions only from energies that are close to each other. The value of $Z(2 \beta)$ therefore sets the height of the universal "plateau" feature, and the approach to it, the "ramp," has its size set by how rapidly the energy correlations die away at large $t$. The "dip" region is formed by the process of handing over from the decreasing disconnected part to the increasing connected part. The formalism here allows, using the complete package of almost 1000 good wave functions and energies, for this all to be computed to good accuracy for JT supergravity (and for a nonperturbatively well-behaved definition of JT gravity in Sec. VI) for the first time. Using again the JT supergravity example, Fig. 14 shows the disconnected contribution to the spectral density function, for $\beta=50>\beta_{\text {cr }}$, with $\log _{10}$ axes.

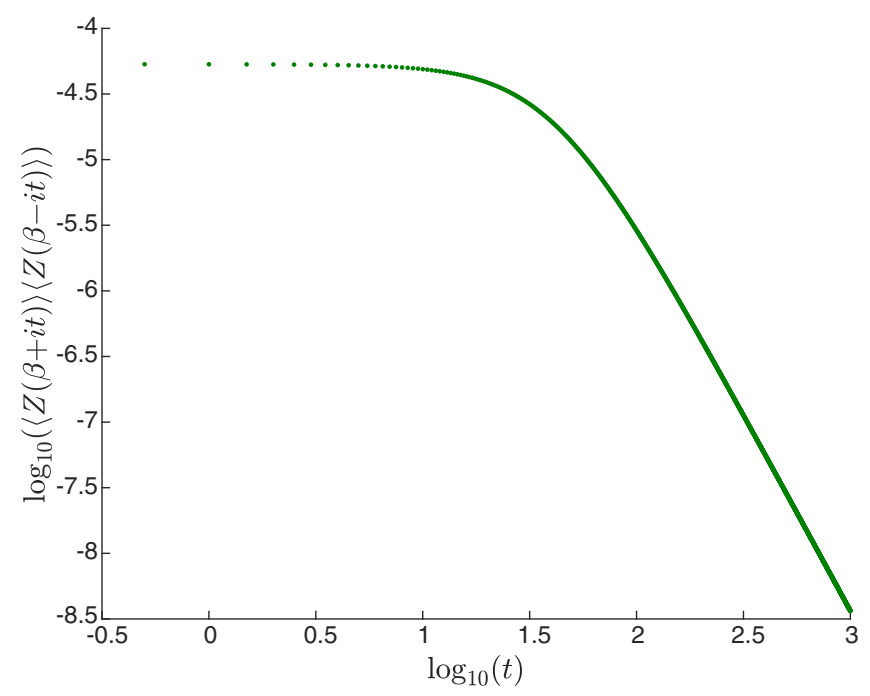

FIG. 14. The disconnected part of the $(2,2)$ JT supergravity spectral density function vs $t$, at $\beta=50$ and $\hbar=1$, showing the classic slope feature.
The slope behavior is quite evident. Since the axes are logarithmic, it is easy to see from the figure that the slope of it is roughly -3 , suggesting a falloff of $\sim t^{-3}$. This might seem surprising, since it is similar to the perturbative falloff rate for ordinary JT gravity (see Ref. [31]). The reason for this faster rate is clear, and again attributable to nonperturbative physics. The slope's falloff is controlled by the behavior of the end point of the spectral density. While at the disc level for JT supergravity $\rho(E) \sim 1 / \sqrt{E}$, producing a $t^{-1}$ falloff [63], the fact that in the $(2,2)$ case nonperturbative corrections remove this $1 / \sqrt{E}$ behavior results in the faster falloff more usually associated with the ordinary JT case (and Hermitian matrix models). There are far fewer states in the vicinity of the end point. This reasoning predicts that for the $(0,2)$ supergravity, the slope should be closer to a $t^{-1}$ falloff. Indeed, this is clear from the behavior of the disconnected piece for $(0,2)$ supergravity, shown in Fig. 15, for $\beta=50$. There, the slope of the linear part shows $t^{-1}$ falloff. Continuing in line with these expectations is a computation of the same quantity for the $\hbar=1 / 5$ case mentioned earlier. The spectrum was shown in Fig. 12, and from there it is natural to guess that the falloff would be even faster, since the nonperturbative effects have scooped away even more states near the end point. A check showed that this is indeed correct, although another figure will not be presented to display the result, to save repetition.

Moving to the connected contribution's time dependence, the $(2,2)$ case is shown in Fig. 16 for the same value of $\beta=50$. The ramp and plateau structures, and the transition between them, are visible. Strikingly, the rise to the plateau is very short lived, the ramp regime rising only a small (on the logarithmic scale) amount before transitioning to the plateau.

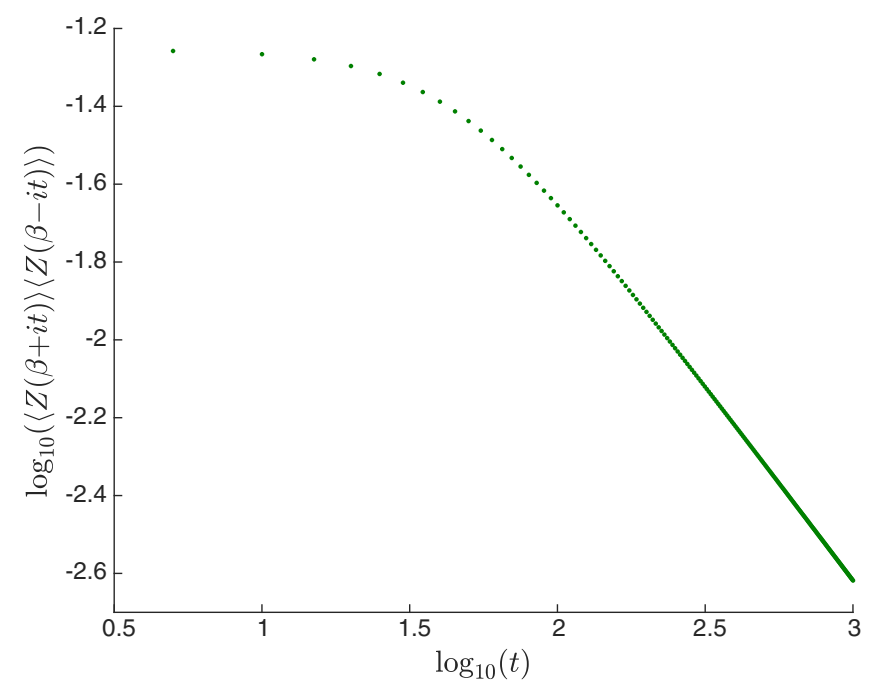

FIG. 15. The disconnected part of the $(0,2)$ JT supergravity spectral density function vs $t$, at $\beta=50$ and $\hbar=1$. 


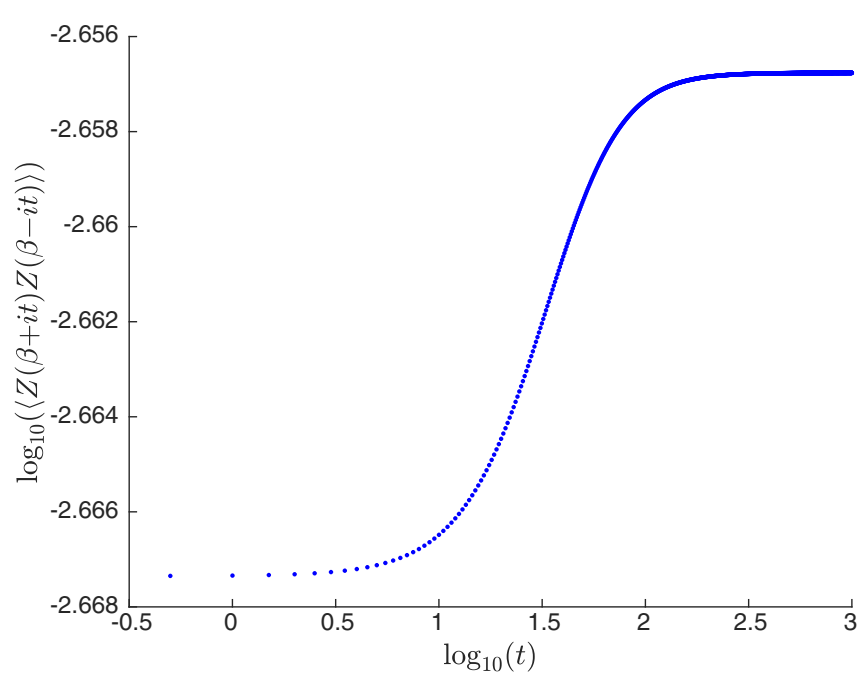

FIG. 16. The connected part of the $(2,2)$ JT supergravity spectral density function vs $t$, at $\beta=50$ and $\hbar=1$, showing the classic ramp and plateau features.

The beginning shape of the ramp is already anticipated in the perturbative answer, $\sim \beta^{-1} \sqrt{\beta^{2}+t^{2}}$, long known for two-macroscopic-loop correlators [44] (appropriately continued to yield the $t$ dependence [5]), but there are strong nonperturbative corrections such that before the long-time linear part can manifest, the other effects turn the ramp over into the plateau.

A similar story is told, initially, by the ramp shape seen for the $(0,2)$ case, shown in Fig. 17 (again for $\beta=50$ ). However, there is a new feature. The rise is indeed slow, but it is remarkably slow. After almost 2 orders of magnitude more time has elapsed, as compared to the $(2,2)$ case, the saturation to the plateau has still not quite completed.

This is a rather novel feature of this case, and worth further investigation. The origin of this physics is most

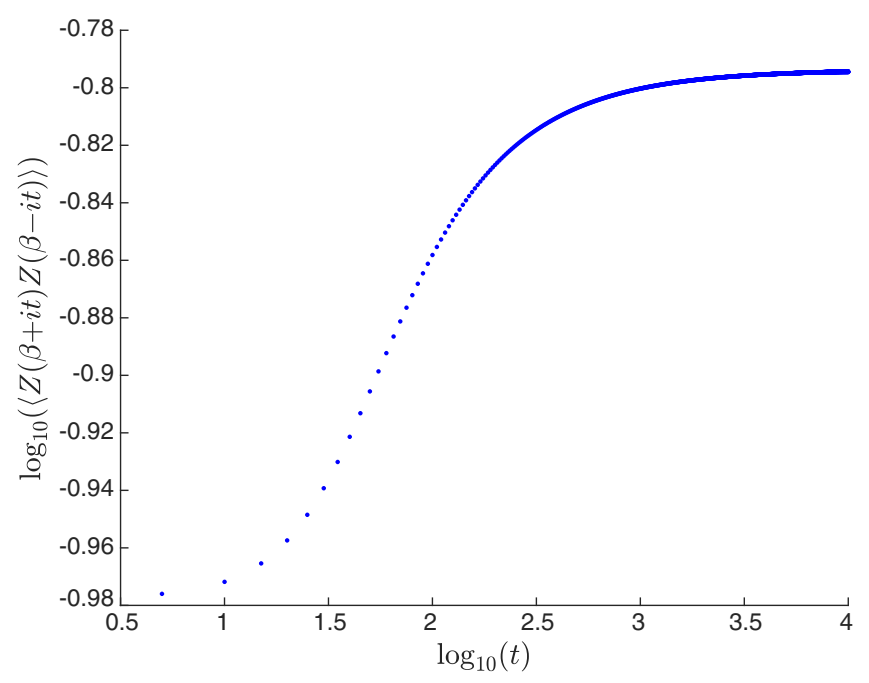

FIG. 17. The connected part of the $(0,2)$ JT supergravity spectral density function vs $t$, at $\beta=50$ and $\hbar=1$. likely again to be attributed to the peculiar pileup of states in the vicinity of $E=0$ that this model has. There is an endless supply of closely spaced low-lying states contributing to the part of the form factor that subtracts from the saturation value $Z(2 \beta)$ [see Eq. (26)]. At longer and longer times, there are even more low-lying states to contribute, and still more closely spaced, maintaining their effect of slowing the saturation.

Turning back to the ramp itself, note that this was [for both $(2,2)$ and $(0,2)]$ for the case $\hbar=1$. At smaller values of $\hbar$, there is more time for the ramp to develop, with an increased rate of rise before the turnover. Note, however, that it is only for extremely small $\hbar$ that the linear behavior of the ramp has a chance to appear.

There are two important lessons here. The first is that associating the ramp with linear behavior (as is sometimes done in the literature) is maybe not the most accurate descriptor. The second is that nonperturbative effects can enhance the appearance of the ramp in the JT supergravity case (in the full spectral form factor made by taking the sum of disconnected and connected parts), even though perturbative expectations might have suggested a reduction [63]. The potential reduction of the ramp feature is based on the idea that a slow $t^{-1}$ rate of fall might not give the ramp time to develop before the plateau sets in. In fact, nonperturbative effects are seen here to produce a rapid fall (sometimes faster even than the perturbative bosonic $t^{-3}$ ), giving plenty of time to develop a sharp "dip", a clear ramp, and a smart turnover into the plateau for the $(2,2)$ case.

The sum of the connected and disconnected pieces gives, for $(2,2)$ supergravity, the classic saxophone shape ${ }^{10}$ known from studying spectral density functions in a wide range of contexts. It is displayed in Fig. 18. This is for $\hbar=1$, and the case of $\hbar=1 / 5$ was already presented in Fig. 1, at $\beta=35$. The latter, being at smaller $\beta$ and $\hbar$, is larger overall and develops a wider variation. The linear part of the ramp would be even more visible for lower values of these parameters.

For $(0,2)$ JT supergravity, Fig. 19 shows the resulting $\hbar=1$ full spectral form factor, again at $\beta=50$. It is, as is to be anticipated, almost 2 orders of magnitude larger than for the $(2,2)$ case, because of nonperturbative effects (already discussed). There is (on the logarithmic scale) a ramp-toplateau transition [although it is, from the discussion above, much slower than for $(2,2)]$. Also visible is the slower slope-to-dip time (due to its slower decay rate), as already discussed.

\section{Temperature dependence}

It is of interest to see how the spectral form factor evolves as a function of temperature. The results for a series of

\footnotetext{
${ }^{10}$ The shape deserves a name, and saxophone seems a good choice, to balance out the many uses of the name "trumpet" in other aspects of JT gravity.
} 


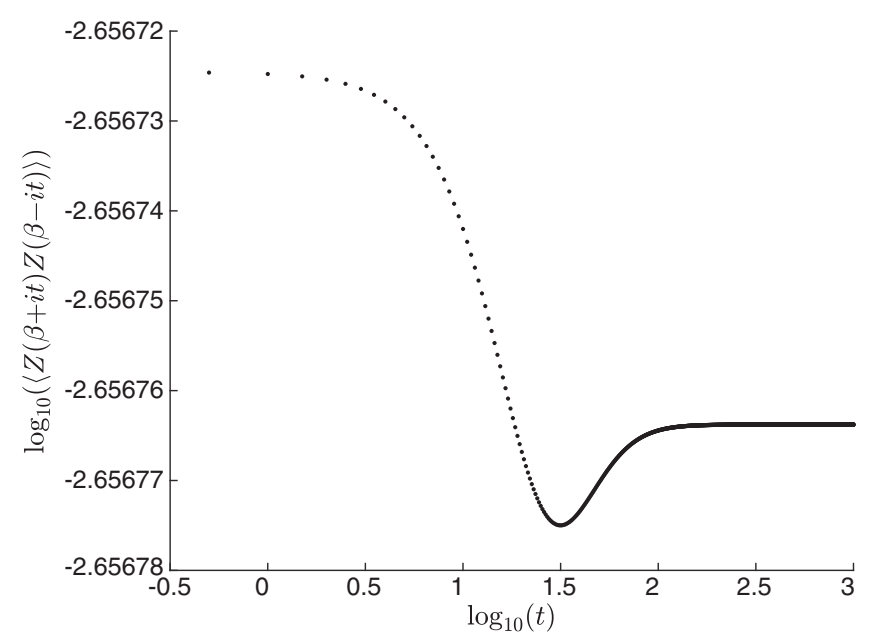

FIG. 18. The full spectral form factor for the $(2,2)$ model of JT supergravity at $\beta=50, \hbar=1$. (The case of $\hbar=1 / 5$ is in Fig. 1).

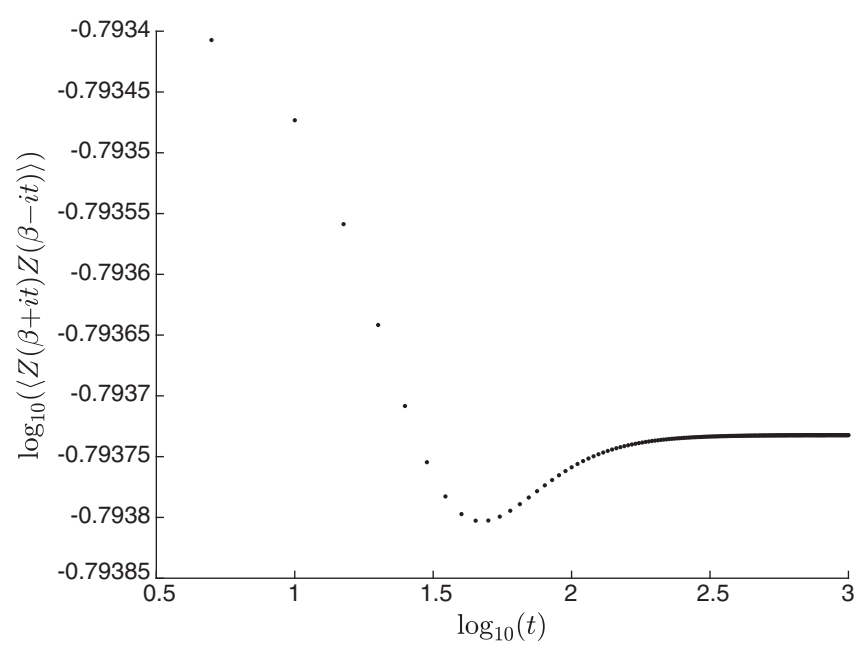

FIG. 19. The full spectral form factor for $(0,2)$ JT supergravity. Here, $\beta=50$ and $\hbar=1$. Cf. the $(2,2)$ case in Fig. 18 .

increasing temperatures, $\beta=50,46,42,38,34$, and 30 , are presented for the disconnected part of the spectral density in Fig. 20.

The highest-temperature curve is at the top. Strikingly, the curves soon merge into each other and follow the falloff already discussed, regardless of the starting temperature. In Fig. 21, there is a series of the full spectral density, for the same set of temperatures. Crucially, for comparison purposes, the curves are all uniformly scaled (on the vertical axis) to have the same initial height as the highest-temperature $(\beta=30)$ case, which is the lowermost curve. Therefore, in this figure, relative slopes should not be taken literally. This scaling allows for ready access to some of the more meaningful comparisons to be made, such as the relative sizes of the curves: Higher-temperature (smaller $\beta$ ) gives a vertically larger curve: Higher temperature "shakes up" the system more, resulting in a wider

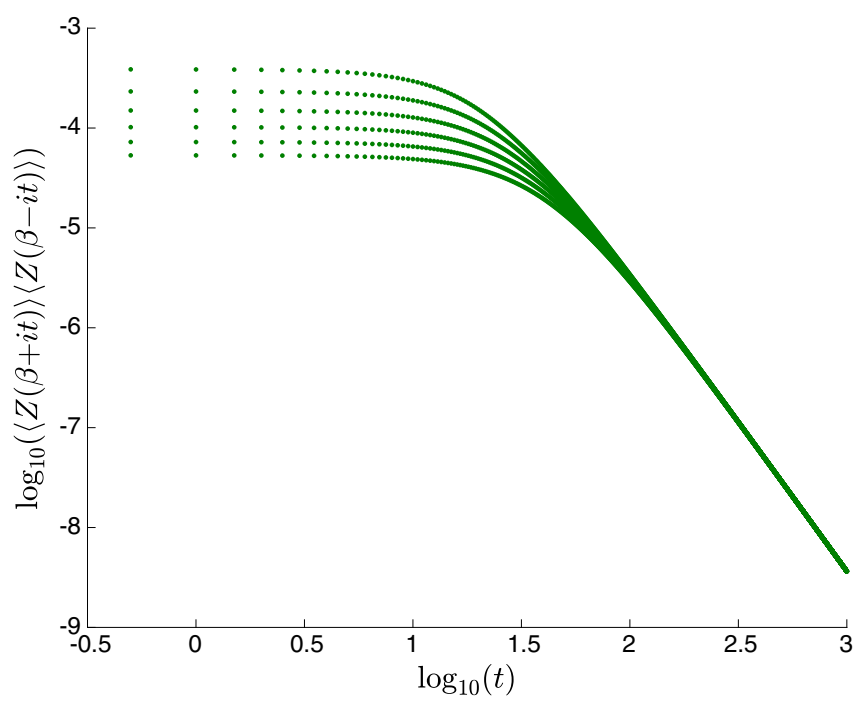

FIG. 20. A series showing the evolution, with $\beta$, of the disconnected part of the spectral form factor, for $(2,2)$ JT supergravity. Here $\hbar=1$.

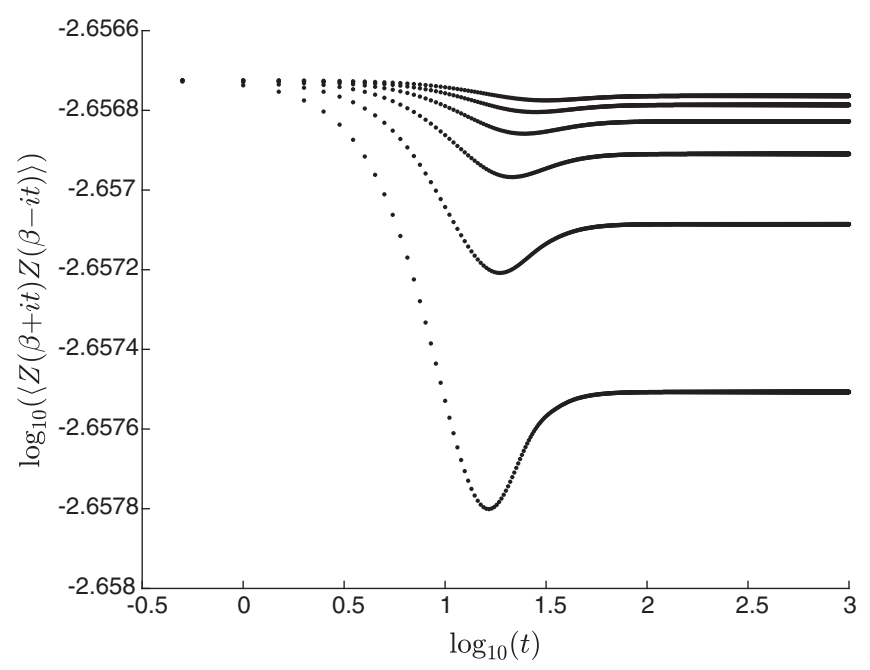

FIG. 21. A series showing the evolution, with $\beta$, of the full spectral form factor, for $(2,2) \mathrm{JT}$ supergravity. Here, $\hbar=1$. For comparison, the curves have been rescaled to start out at the same height as the highest-temperature curve.

amplitude of deviation from the initial value before it settles down. Interestingly though, the dip time increases slightly with higher temperature, although not dramatically. The rapid ramp time also changes very slowly with $\beta$.

Heading toward smaller (but still moderate) values of $\beta$ (in the region of $\beta \simeq \beta_{\text {crit }}$ ), there are small modulations in both the disconnected and connected parts of the form factors, accumulating (in the latter) near the crossover from ramp to plateau. The combined result of these highertemperature structures is that there is a damped wobble as the ramp merges into the plateau. An example of the full spectral form factor showing this feature is given in Fig. 22 


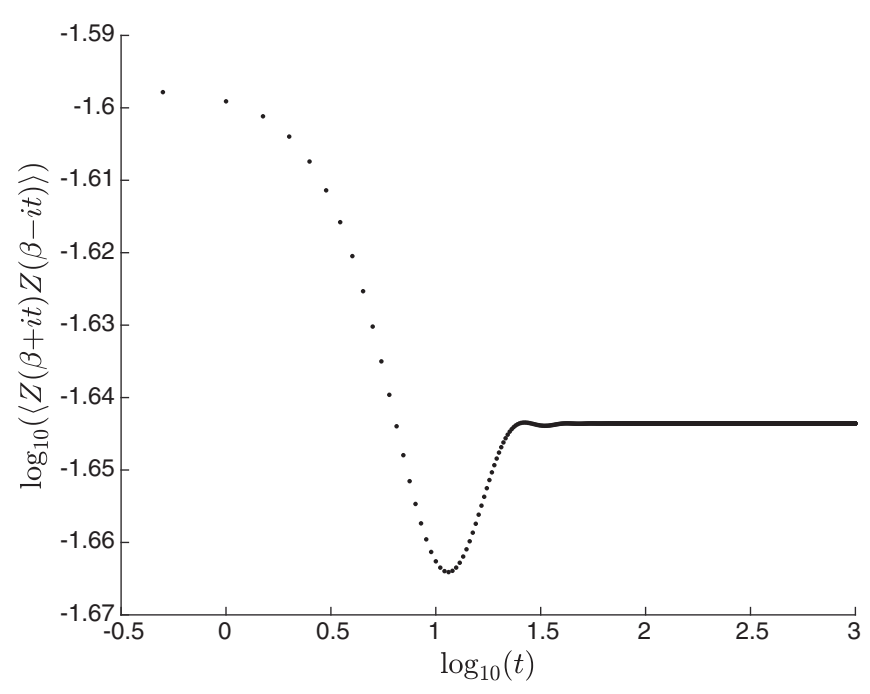

FIG. 22. The full spectral form factor at $\beta=14, \hbar=1$, for $(2,2)$ JT supergravity. A (relatively) high-temperature feature appears near the crossover from ramp to plateau. See text for discussion.

for the case $\beta=14$. Whether this is interesting physics or not is not clear. ${ }^{11}$ The value of the temperature at which this can be seen seems comfortably below the highest energy allowed by the truncation.

Some other fascinating structures become apparent in the very high-temperature regime. How useful they are for the physics in question is debatable, since this whole context (the Schwarzian, the connection to black holes, SYK, etc.) is in a low-energy limit. Moreover, high temperature also begins to go beyond the energies for which the truncation of the string equation remains reliable. However, it is interesting to observe the features anyway, and could well be instructive for understanding JT models with a cutoff [65-68]. Looking at the disconnected part of the spectral form factor for the $(2,2)$ supergravity case, a series of dips evolve, becoming more pronounced toward higher temperature (smaller $\beta$ ). See Fig. 23.

They have a clear pattern and structure and are consistent with observations made for large- $N$ random matrix systems (even without double scaling). (They are also analytically obtainable in the exact Airy example reviewed in Appendix A.)

In fact, the disconnected function is beginning to resemble the form $J_{1}(t)^{2} / t^{2}$ that has been derived analytically for the infinite-temperature case. After taking the logarithm, the zeroes of the Bessel function $J_{1}$ become the dips in the logarithmic plot. It would be interesting to show that this analytical form (or a variation thereof) emerges in this JT supergravity context as well. Following the

\footnotetext{
${ }^{11}$ It is reminiscent of features of an interesting exact expression derived in Ref. [64] in the context of an SYK model with source terms.
}

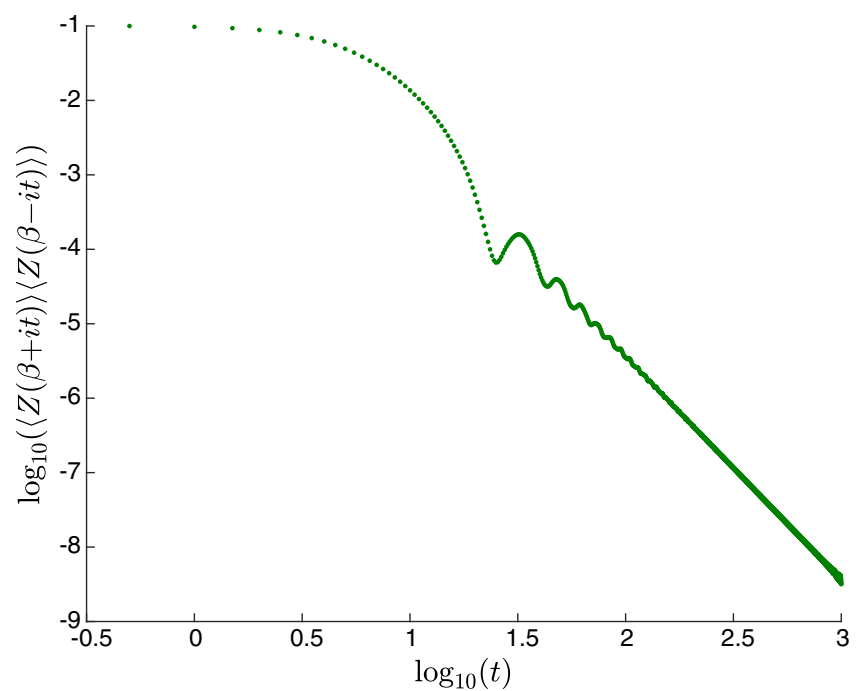

FIG. 23. Disconnected part of the spectral form factor at $\beta=9$, $\hbar=1$, for $(2,2)$ JT supergravity. The growing dips are consistent with a pattern of zeros developing at infinite temperature.

numerics to smaller $\beta$ seems to confirm this (a zero in the connected function also appears, in some examples), although eventually numerical inaccuracies begin to overwhelm the results, presumably because the correct physics needs to include contributions from energies that lie beyond the cutoff on the spectrum up to which the truncated equations are valid.

\section{NONPERTURBATIVE JT GRAVITY}

This section presents results analogous to those shown in earlier sections for the nonperturbative completion of ordinary JT gravity presented in Ref. [3]. It might seem odd to have studied the JT supergravity examples first, leaving this case for last, but there is good reason. The nonperturbative physics of this case is more subtle. JT gravity was shown, in Ref. [5], to be perturbatively (in the topological expansion) equivalent to a double-scaled Hermitian matrix model-i.e., classified in the Gaussian unitary ensemble ( $\beta=2$ in the Dyson-Wigner series). On general grounds, such double-scaled Hermitian matrix models are known to sometimes have nonperturbative (in topology) instabilities, and so it is possible that the JT gravity definition inherits them. More specifically, thinking about the model in terms of constituent minimal models, as in Sec. III, it is made up of an interpolating family of minimal models that have the $x \rightarrow-\infty$ boundary condition in Eq. (12) in both directions, and instead solve the string equation $\mathcal{R}=0$. [Recall that $\mathcal{R}$ is given in Eq. (10)]. These are the $(2 k-1,2)$ bosonic minimal string models. For even $k$, these models are nonperturbatively unstable, as has been known for some time [46,69-71]. From the point of view of the spectrum, all the models, when nonperturbative effects are taken into account, have 
contributions from arbitrarily negative energy sectors. Even though exponentially suppressed, for even $k$, the effective potential turns downward for states at these energies, signaling the system's wish to tunnel to an entirely new solution that is quite different from the one around which perturbation theory was developed. From the perspective of this paper (solving string equations nonperturbatively), this means that for each of those ( $k$-even) models, there simply are no real smooth solutions of the $k$ th equation with those conditions $[46,70,72,73]$. Since JT gravity is made up of, in equal measure, even and odd $k$ models, this strongly suggests that it inherits these problems, as already noted in Ref. [5]. ${ }^{12}$

The route that Ref. [3] took to supply a nonperturbative definition of JT gravity was to embed it into a larger problem. The minimal models used in previous sections for JT supergravity, also indexed by $k$, have the same $x \rightarrow-\infty$ boundary condition as the bosonic minimal models, and in fact when $\Gamma=0$, they have identical perturbation theory to solutions to the differential equation (9). Put differently, they solve $\mathcal{R}=0$ perturbatively at large $-x$. This means that if used to construct a JT gravity model, they will yield the same physics at high energies $E$, but yield different physics at lower energies that is untroubled by the stability issues. The combination of models needed (that will give the Schwarzian spectral density [Eq. (4)]) at high $E$ is as follows:

$$
t_{k}=\frac{1}{2} \frac{\pi^{2 k-2}}{k !(k-1) !}
$$

(This relation was first derived in Ref. [12], but with different normalization.) In fact, it is possible to integrate the $f\left(u_{0}\right)$ that results from this combination to find the explicit classical potential $u_{0}(x)$ that yields the Schwarzian density, through

$$
x=-\frac{\sqrt{u_{0}}}{\pi} I_{1}\left(2 \pi \sqrt{u_{0}}\right)
$$

the analogue of the case (15) for the JT supergravity found earlier. Then the $u(x)$ for JT gravity is constructed fully nonperturbatively using Eq. (9) with $\Gamma=0$. This nonperturbative taming of JT gravity can be explored extensively along the same lines as was done for the JT

\footnotetext{
${ }^{12}$ An earlier version of this manuscript contained an idea for a possible evasion of this reasoning. Since individual odd- $k$ members of the $(2 k-1,2)$ minimal series are better nonperturbatively defined, the idea was that perhaps one could define the model as an odd- $k$ model (for large $k$ ) within which all the lowerorder models are turned on. This was thought to possibly yield good nonperturbative solutions to the $\mathcal{R}=0$ differential equation, as deformations of the known good solutions for odd $k$. This does not seem to work.
}

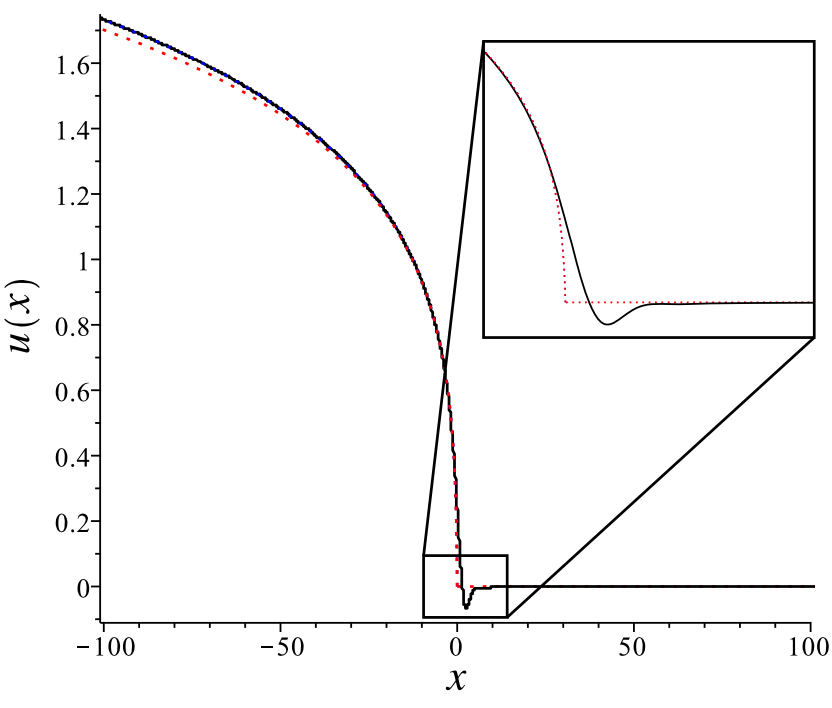

FIG. 24. The solution (solid line) of the string equation for truncation up to $t_{7}$. The inset shows the well that develops in the interior. The full classical solution is shown too (dotted line).

supergravity models. The truncation scheme works similarly, and so there is no need to retread the ideas again.

A solution to the string equation [Eq. (9)] with the bosonic JT combination of minimal models [Eq. (28)] was found with the first seven models turned on, constituting a very good truncation where energies up to $E \sim 1.5$ can be trusted. A combination of numerical and analytic methods was used to find the solution to the 15th-order differential equation. See Sec. I of Appendix B for some tips on how this was done. Figure 24 shows the solution, with the classical (disc-level) potential that gives the JT spectral density [Eq. (4)], displayed as a dashed line.

Again, there is a small well (not deep enough to support bound states) in the interior, and then $u(x)$ settles to zero at the right. These are features shared by the JT supergravity models, as should be expected, since their components are being used here as a nonperturbative low-energy "regulator" in a sense, removing the leakage to negative energy.

The methods of Sec. IV then allow for the spectrum to be computed. For this definition, $\mu=0^{-}$is used for the upper limit in Eq. (21), matching the $x<0$ perturbation theory that builds JT gravity (see the discussion in Ref. [4] for more discussion about how to efficiently extract perturbation theory using the Gel'fand-Dikii resolvent equation), so in contrast to the JT supergravity cases, there is no $1 / \sqrt{E}$ classical contribution to the spectrum. Figure 25 displays, for the first time, the spectral density of a nonperturbative completion of JT gravity with all perturbative and nonperturbative corrections included (up to this energy). It is clear from the figure that the nonperturbative ripples have already begun to die away and merge into the classical smooth region, showing that this truncation has captured the key physics that is affected by 


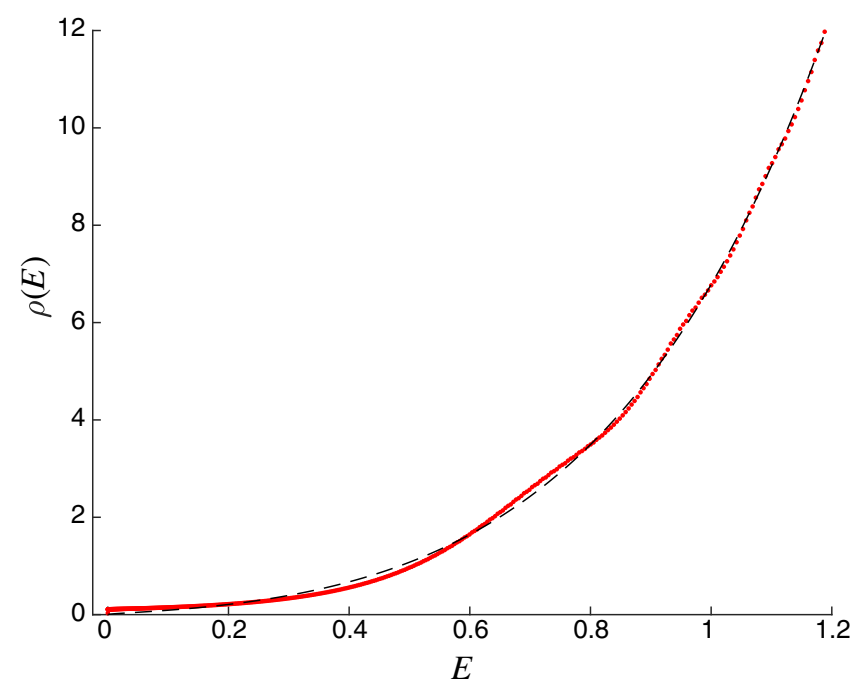

FIG. 25. The full spectral density, for the nonperturbative completion of JT gravity in Ref. [3]. The dashed blue line is the disc-level result of Eq. (4).

nonperturbative contributions. Notice that the spectrum is naturally bounded below by $E=0$, as was already shown explicitly in preliminary studies in Ref. [3]. Note that Ref. [3] generalized the construction by turning on the parameter $\mu$ that was discussed in the supergravity context in Sec. IV. This is straightforward to do, and there were no additional insights to be gained, and so results are not presented here. The tail of the resulting distribution, not surprisingly, resembles the tails already displayed in Ref. [3], including the interesting feature that nonperturbative effects generate a nonzero $\rho(E=0)$.

Of course, with the spectrum in hand (approximately 1800 normalized wave functions and their energies), the next natural step is to compute the spectral form factor,

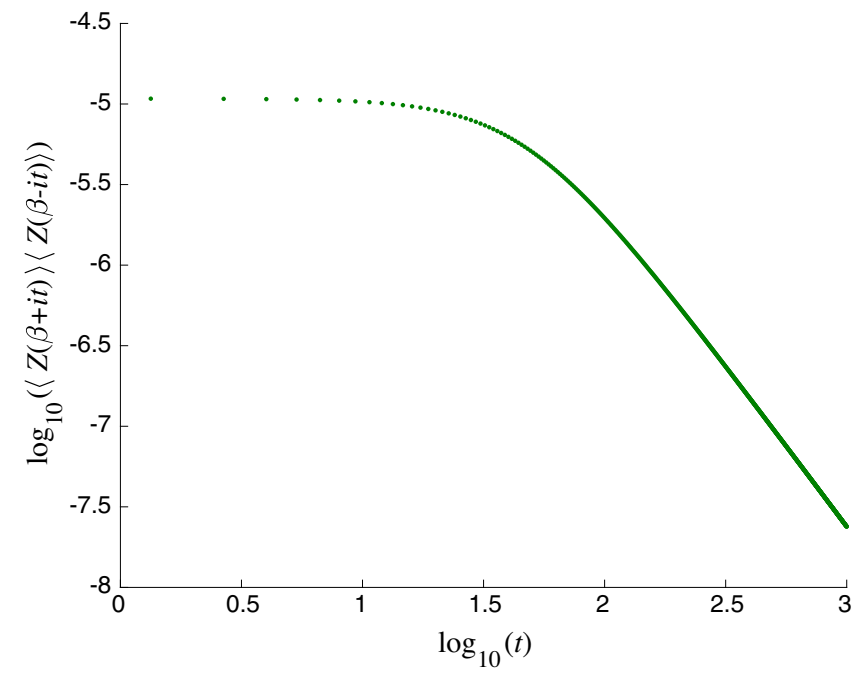

FIG. 26. The disconnected part of the JT gravity spectral form factor vs $t$, at $\beta=50$ and $\hbar=1$, for the nonperturbative scheme of Ref. [3].

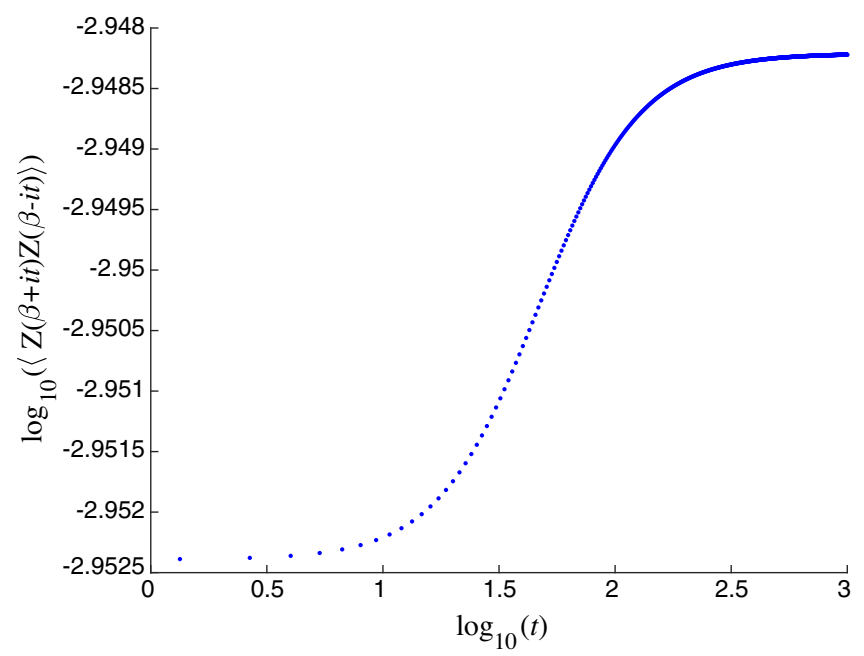

FIG. 27. The connected part of the JT gravity spectral form factor vs $t$, at $\beta=50$ and $\hbar=1$, for the nonperturbative scheme of Ref. [3].

using the methods of Sec. V. The correlator of two boundaries is readily computed, and the phase transition where the disconnected part (two black holes) hands over to the connected part (wormhole) happens at $\beta_{\mathrm{cr}}$. (A figure similar to Fig. 13 is omitted here to avoid repetition.) For $\beta=50$, the disconnected, connected, and combined spectral form factors are shown in Figs. 26, 27, and 28, respectively.

The most striking feature overall is in the disconnected portion of the form factor, controlling the initial slope. As might be expected from the absence of the $1 / \sqrt{E}$ lowenergy behavior of the regulating models, the time dependence of the slope is not $t^{-1}$ [as it is for the $(0,2) \mathrm{JT}$ supergravity], but neither is it the $t^{-3}$ expected from the classical low-energy physics to be read off from the $\beta^{-3 / 2}$

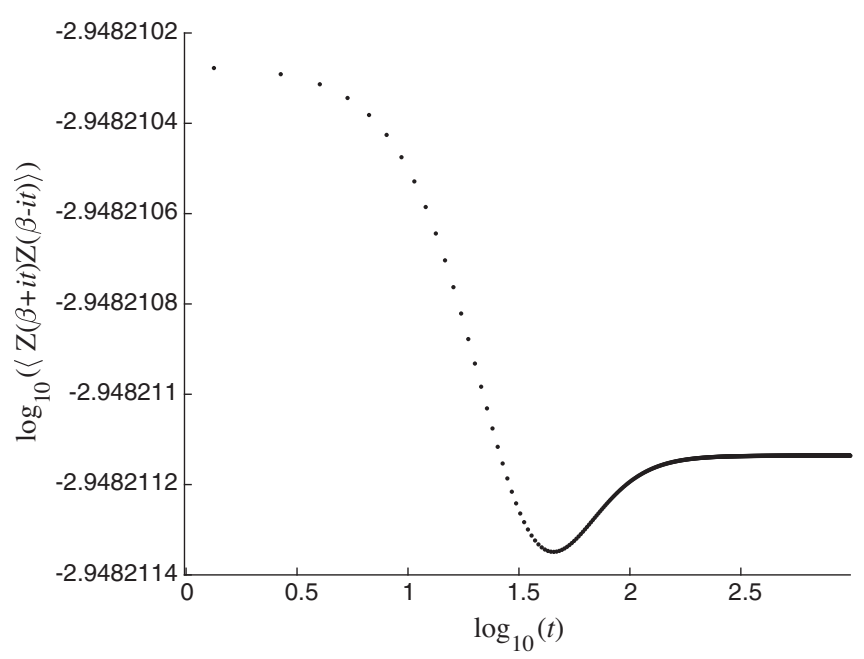

FIG. 28. The full JT gravity spectral form factor vs $t$, at $\beta=50$ and $\hbar=1$, for the nonperturbative scheme of Ref. [3]. 
dependence of the partition function [Eq. (2)]. Rather, it interpolates between them, and is $\sim t^{-2}$, to the nearest integer. From what was learned from the supergravity cases of the previous section, the origin of this is clear. The end point of the distribution has a different structure (see Fig. 25), with some nonzero $\rho(0)$ at the end. There are far fewer states than for the $(0,2)$ supergravity case, but more than for the $(2,2)$ case, and hence the falloff rate (at least for this value of $\hbar$ ) is between that of those two cases.

Again, as is seen in all the models studied in this paper (and also the special Airy model recalled in Appendix A), nonperturbative effects hasten the transition from dip to ramp to plateau such that the linear part of the classical contribution to the ramp that emerges at long times simply does not have time to develop for moderate values of $\hbar$.

\section{CLOSING REMARKS}

The purpose of this paper was to explicitly uncover and examine the nonperturbative physics for JT gravity and supergravity that is accessible if they are formulated using minimal model building blocks. This construction is not a simple large- $k$ limit of a minimal model, but a more refined affair involving coupling them together in a particular combination, as suggested perturbatively in Ref. [12], and extended to nonperturbative physics in Refs. [3,4]. The principal applications that demonstrated the facility of the technique were the explicit computation, for the first time, of the full nonperturbative spectral densities of various JT gravity and supergravity models, and the use of these spectral densities to compute the spectral form factor in each case, showing how the nonperturbative effects affect the shape (sometimes dramatically) of this important diagnostic quantity. Having explicit access to the nonperturbative features in this manner turned out to be rather instructive, as extensively discussed in the body of the paper.

Techniques to allow such nonperturbative properties to be extracted, in a consistent and well-defined scheme (for generic values of $\beta$ and $\hbar$ ), have not been presented in the literature before, and it is hoped that these methods and results will go some way toward helping uncover more of the fascinating web of interconnections between geometry, quantum mechanics, gravity, and chaos that seems to be emerging from these studies.

It is likely that other models of JT gravity can be "deconstructed" in terms of minimal models in a way analogous to what was done here, and thereby can be given a nonperturbative definition, not just in principle, but (as shown here) in useful accessible terms. It could possibly also encompass some of the new kinds of matrix model descriptions of JT-gravity black holes mentioned recently in Refs. [74,75]. Perhaps the results of explorations along these lines will be reported soon.

\section{ACKNOWLEDGMENTS}

C. V. J. thanks Felipe Rosso and for helpful questions and comments, Douglas Stanford and Edward Witten for helpful questions, the U.S. Department of Energy for support under Grant No. DE-SC 0011687, and, especially during the pandemic, Amelia for her support and patience.

\section{APPENDIX A: NUMERICAL TESTBED: THE AIRY MODEL}

As a means of sharpening understanding of some of the key features of the spectral form factor, and for modeling what kinds of physics can be reliably captured by the numerical approaches used in the main body of the paper, this appendix presents a numerical exploration of the exactly solvable Airy model, which is the double-scaled limit of the simple Hermitian matrix model with Gaussian potential, obtained by magnifying the infinitesimal region at the edge of Wigner's semicircle [44]. It is the $k=1$ model of the $(2 k-1,2)$ minimal string series, and as such is also a model of the extreme low-energy tail of the matrix model of JT gravity in Ref. [5].

In the language of this paper, it comes from using the simple linear potential $u(x)=-x$ in the Hamiltonian [Eq. (7)], and the resulting equation to solve for the spectrum is simply (after a change of variables) Airy's differential equation. The wave functions for energy $E$ are

$$
\psi(E, x)=\hbar^{-\frac{2}{3}} \operatorname{Ai}\left(-\hbar^{-\frac{2}{3}}(E+x)\right),
$$

and the spectral density that results is

$\rho(E)=\int_{-\infty}^{0}|\psi(E, x)|^{2} d x=\hbar^{-\frac{2}{3}}\left[\operatorname{Ai}^{\prime}(\zeta)^{2}-\zeta \operatorname{Ai}(\zeta)^{2}\right]$,

where $\zeta=-\hbar^{-\frac{2}{3}} E$. See Fig. 29 for a plot of the spectrum, showing the exponential tail running to negative $E$. At large $E$, the nonperturbative oscillations of the Airy function die out, leaving the classical (disc) contribution $\rho_{0}(E)=(\pi \hbar)^{-1} \sqrt{E}$. This is shown as a dashed line.

The correlator of two boundaries can be computed exactly using properties of the Airy functions out of which the wave function is built. ${ }^{13}$ The disconnected part is simply the square of the partition function, which can be evaluated by Laplace transform, remembering to include negative energies to incorporate the full nonperturbative spectrum:

$$
Z_{\mathrm{Ai}}(\beta)=\int_{-\infty}^{+\infty} \rho_{\mathrm{Ai}}(E) e^{-\beta E} d E=\frac{e^{\frac{\hbar^{2}}{12} \beta^{3}}}{2 \pi^{1 / 2} \hbar \beta^{3 / 2}} .
$$

This gives

\footnotetext{
${ }^{13}$ In fact, Ref. [76] writes down expressions for correlators of multiple loops in this model.
} 


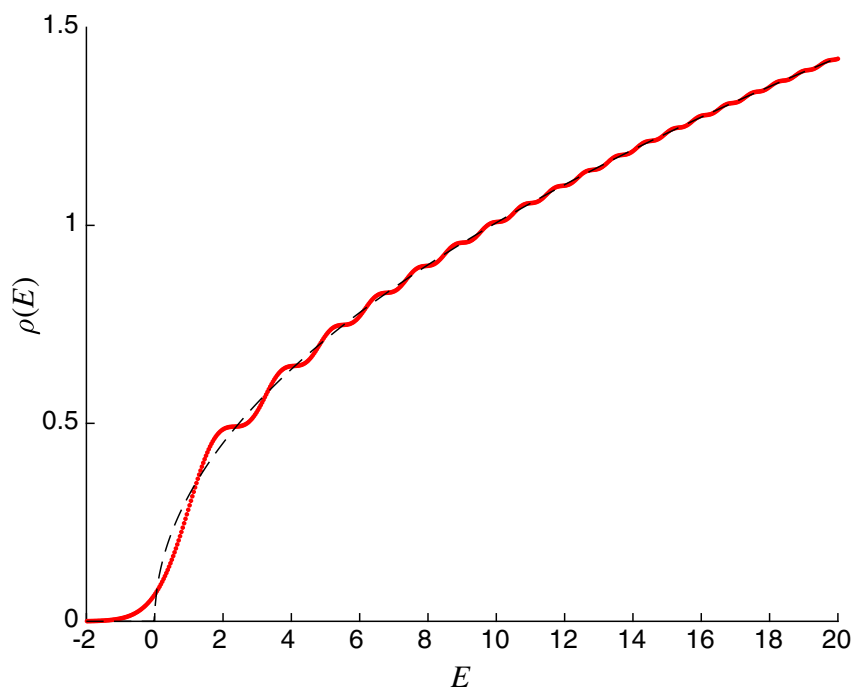

FIG. 29. The full spectral density for the exactly solvable Airy model, at $\hbar=1$. The dashed line is the disc-level result.

$$
\langle Z(\beta)\rangle\left\langle Z\left(\beta^{\prime}\right)\right\rangle=\frac{e^{\frac{\hbar^{2}}{12}\left(\beta^{3}+\beta^{\prime 3}\right)}}{4 \pi \hbar^{2}\left(\beta \beta^{\prime}\right)^{3 / 2}},
$$

while implementing Eq. (26) yields the connected piece to be

$$
\left\langle Z(\beta) Z\left(\beta^{\prime}\right)\right\rangle=\frac{e^{\frac{\hbar^{2}}{12}\left(\beta+\beta^{\prime}\right)^{3}}}{2 \pi^{1 / 2} \hbar\left(\beta+\beta^{\prime}\right)^{3 / 2}} \operatorname{Erf}\left(\frac{1}{2} \hbar \sqrt{\beta \beta^{\prime}\left(\beta+\beta^{\prime}\right)}\right) .
$$

These are exact expressions-i.e., both perturbative and nonperturbative parts are incorporated (see Refs. [44,76] and the review in the Appendix of Ref. [12]). Nevertheless, it is instructive to pretend that only a finite number of wave functions are known (only numerically) for a discrete set of energies up to some maximum energy. The question is then how well the exact expressions can be reproduced. This is the situation of the body of the paper, resulting from the controlled truncation of the infinite-order string equation.

The answer to the question, reassuringly, is that a great deal of the important physics is accessible. To show this, a set of 1000 of the wave functions [Eq. (A1)] were discretized on the same size grid used in the body of the paper ( $x$ is broken up into 20000 points), for a range of energies $-20 \geq E \geq 20$, and the same code that performed the numerical implementation of the expressions given in Eqs. (20) and (26) was carried out for this exact model. The result presented in Fig. 30 shows the crossover between the two portions of the correlator as a function of $\beta$, and Figs. 31, 32, and 33 show the disconnected, connected, and combined parts of the spectral form factor, for temperature $\beta=1 / 2$. The dashed lines are the plots of the exact

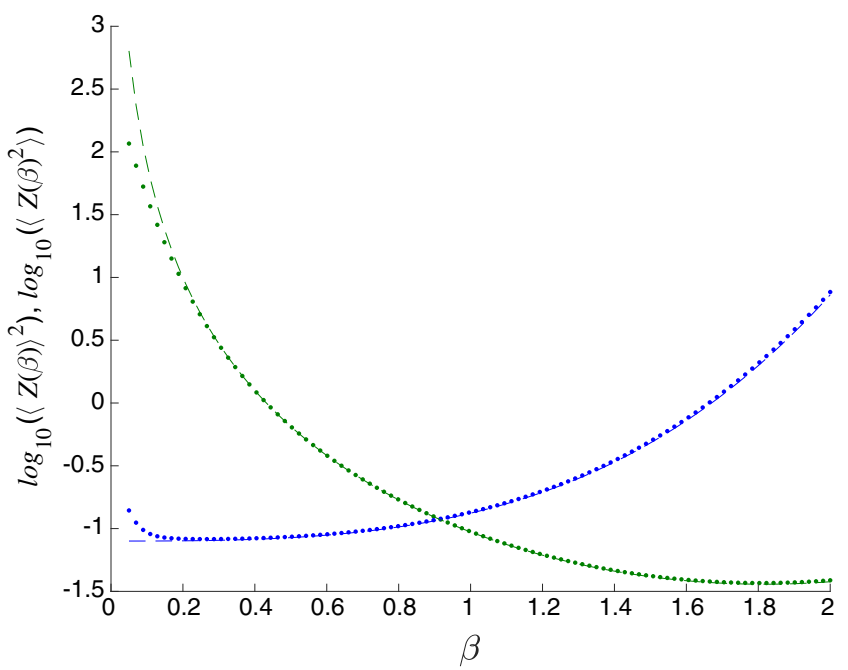

FIG. 30. The disconnected (starting higher at the left) vs the connected (lower) two-point function of the Airy model's partition function as a function of $\beta$, showing a phase transition at $\beta=\beta_{\text {cr }} \simeq 0.92$. A dashed line shows the exact result; the dots are for a numerical truncation.

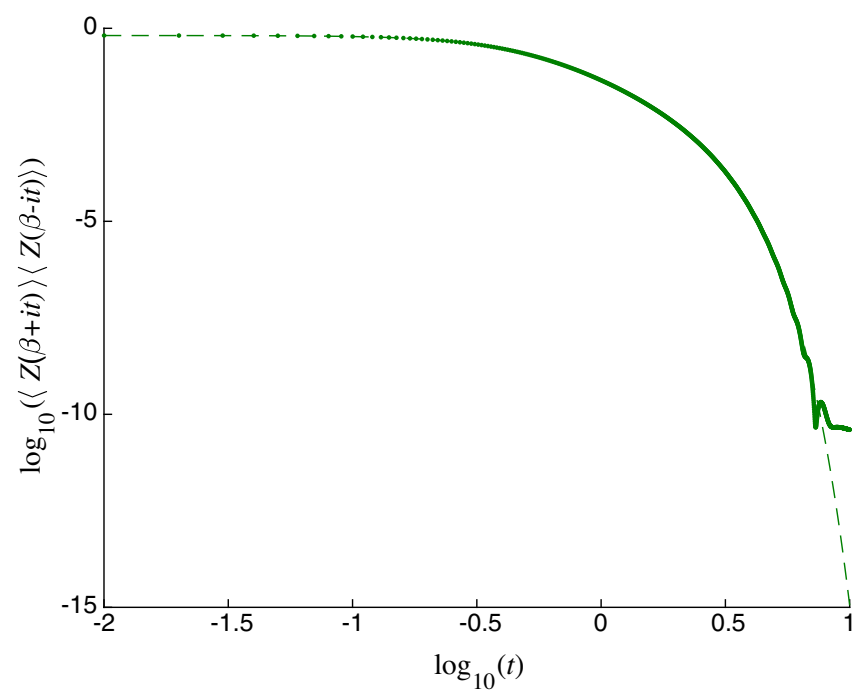

FIG. 31. The disconnected part of the Airy model's spectral form factor vs $t$, at $\beta=1 / 2$, showing the classic slope feature. A dashed line shows the exact result; dots are for a numerical truncation. (The undulations at the end are numerical errors at ultrasmall values, and so should be ignored.)

functions (A4) and (A5), while the dots show the results of the numerical computations.

As might be expected, significant deviations from the dashed lines occur when $\beta$ becomes too small, including patterns of zeros representing finite size effects. This signals temperatures that excite higher energies that are not included in the numerical scheme (but are in the exact expressions), and hence the results deviate. As long as such extremes are avoided (depending upon the truncation 


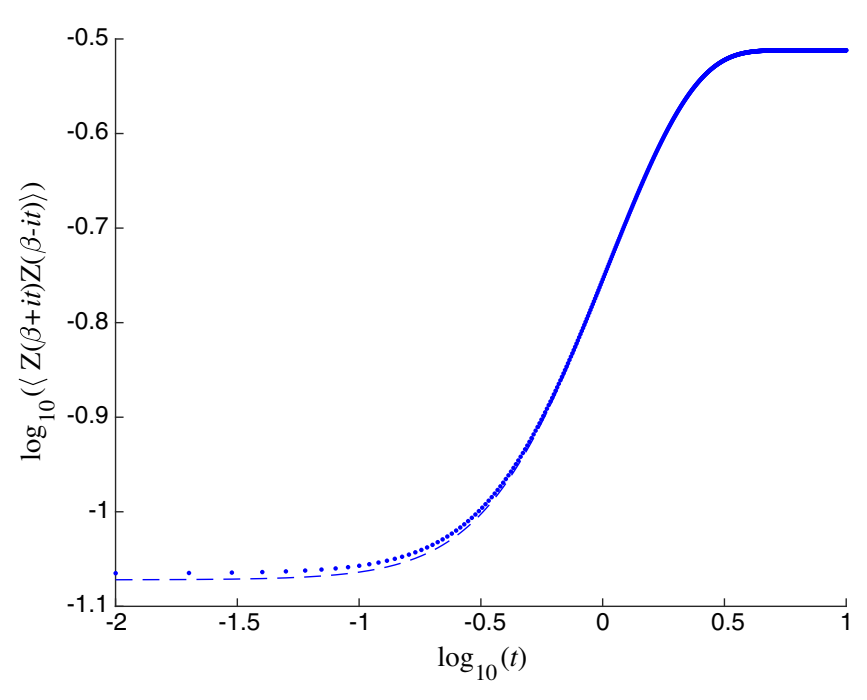

FIG. 32. The connected part of the Airy model's spectral form factor vs $t$, at $\beta=1 / 2$. A dashed line shows the exact result; dots are for a numerical truncation.

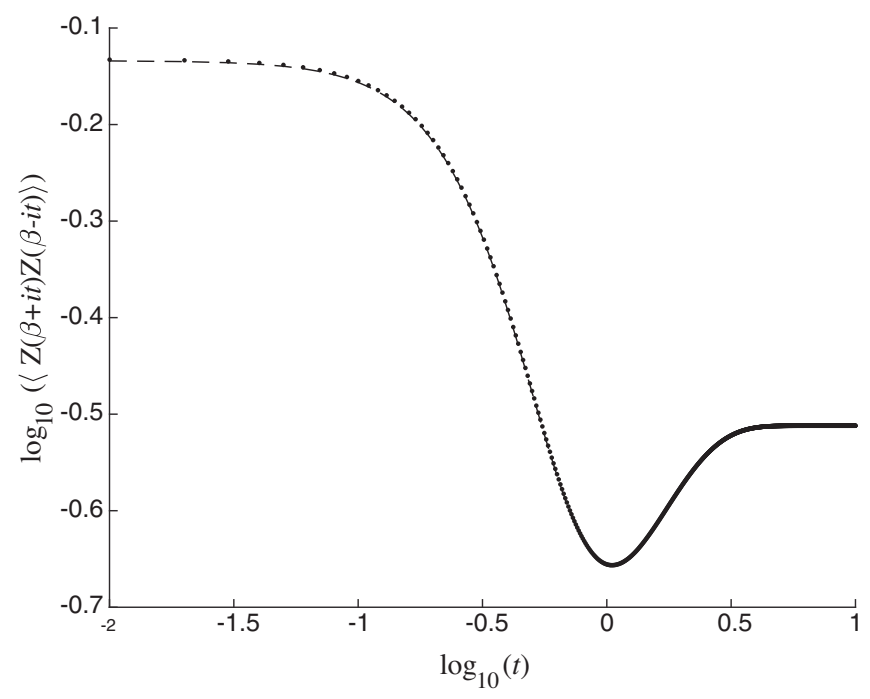

FIG. 33. The full spectral form factor of the Airy model vs $t$, at $\beta=1 / 2$.

energy chosen), the numerical results are very reliable. This is a good controlled model of the truncation scheme used in the body of the paper, showing that the results obtained are robust.

\section{APPENDIX B: NUMERICAL RECIPES}

\section{Suggestions for solving the string equation}

The string equation that supplies the potential $u(x)$ for a particular problem is highly nonlinear, and of high order $(2 k)$ in derivatives if truncating to the $k$ th model. Even the simplest solution [with the boundary conditions of interest given in Eq. (12)], where all $t_{k}$ 's are set to zero except $k=1$, requires numerical techniques to extract its explicit form (it is related to a Painléve transcendent, and hence cannot be written in terms of other elementary functions). Here are some suggestions for finding numerical solutions, to help the interested reader learn how to extract useful information for themselves. First, Maple was used in this case (although MATLAB works well too, as probably would other programs). The dsolve routine was used, with an error tolerance of $10^{-5}-10^{-10}$, depending upon the equation being solved. (In fact, when these equations were first solved [50,77], it was for the cases $k=1,2$, and 3, including cases where the models were nontrivially coupled [78]. Back then, their solution was found by writing a program in FORTRAN that called the routine D02RAF, part of the NAG libraries.)

As mentioned in the text, for various reasons, it makes sense to take an additional derivative of the equation. This reduces the nonlinearity somewhat, at the expense of increasing the order, which is a small price to pay. This is because the first derivative results in an overall factor of $\mathcal{R}$, which can be divided out, since it will not vanish for the solutions of interest. A derivative explicitly removes $\Gamma$ from the equation, however. Now, the only knowledge the system has of the desired choice of $\Gamma$ is through subleading (in the small- $\hbar$ expansion) terms in the boundary conditions, which need to be solved for with all $t_{k}$ 's present. This is nicely organized on the $x>0$ boundary because the presence of the $t_{k}$ 's coming in one by one at successively higher terms in the $1 / x^{2}$ expansion (see Ref. [4]). It is less nice to perform analytically on the $x<0$ boundary. There, all the $t_{k}$ 's contribute at the next order, and solving for the order- $\hbar \Gamma$ correction requires solving a $k$ th-order polynomial. Beyond the $k=4$ truncation, this becomes unpleasant at best. However, if the system is solved on a large enough region, with a small enough discretization, terms beyond the leading left boundary condition can be safely ignored, and a good approximation to the solution can be found anyway. (If needed, however, a recursive code for solving for the subleading corrections to the boundary conditions numerically can be employed.)

This works well for $\Gamma=\frac{1}{2}$, while for $\Gamma=-\frac{1}{2}$ a different technique is used, because the numerical approach is less stable due to a more complicated well shape appearing in the interior, which is hard to control in a 13th-order differential equation. Reference [57] noticed that $\Gamma$ can be changed by an integer using a special "Backlünd" transformation, and actually derived an analytic expression showing how to build the new $u(x)$ at $\Gamma \pm 1$ from the old $u(x)$ (and its derivatives) at $\Gamma$. Here it is:

$$
u_{\Gamma \pm 1}=\frac{3\left(\mathcal{R}^{\prime}\right)^{2}-2 \mathcal{R} \mathcal{R}^{\prime \prime} \pm 8 \hbar \Gamma \mathcal{R}^{\prime}+4 \hbar^{2} \Gamma^{2}}{4 \mathcal{R}^{2}},
$$

[where $\mathcal{R} \equiv \mathcal{R}\left(u_{\Gamma}\right)$, and a sign has been switched to match the current conventions]. So once $u(x)$ for the case of $\Gamma=\frac{1}{2}$ was found using Maple, the output of dsolve contains all 
the derivatives of $u(x)$ needed to construct $u(x)$ at $\Gamma=-\frac{1}{2}$, giving the well structure seen in Fig. 7.

In fact, a similar story held for the case of $\Gamma=0$ used in Sec. VI. There is also a well structure in the interior, which is hard to solve for numerically when at high order and with boundary conditions far from the structure itself. Experience from studying positive-integer $\Gamma$ suggested that $\Gamma=1$ would be smoother to solve for, and that was indeed the case. From there, the transformation (B1) was used to construct the desired $\Gamma=0$ solution.

\section{Suggestions for solving the spectrum}

As mentioned in Sec. IV, in order to solve for the solutions to the eigenvalue problem, $\{E, \psi(E, x)\}$, a matrix Numerov method [58] was used, as it was in Refs. [3,4]. This simply puts the system into a box, and turns the problem into a large matrix diagonalization problem, for a given input potential $u(x)$. This was done using MATLAB. A key point is that it is desirable to have a large number of eigenvalues in the energy range from zero to the chosen highest energy (determined by the level of the truncation of the string equation). So two choices were made to ensure a good set of solutions. The first was to use a large grid, so a grid of $20000 \times 20000$ was used. The second was to use a large box. As stated, the spectrum solving method is essentially putting the system into a box, and a portion of the output eigenvalues and eigenfunctions will be affected by the edges of the box. Those should be discarded, and the larger the box, the more useable eigenstates will be available in the reliable energy window. Since, as already observed in Sec. III, the solution for $u(x)$ becomes similar to the disc-level behavior far away enough from the central region, the box can be easily made larger by connecting the solution (solved numerically out to $-200 \leq x \leq+200$ ) to a wider region [e.g., $-2645 \leq x \leq+2645$ for the $(2,2)$ and $(0,2)$ models], where just the exact disc solution $u_{0}(x)$ is used. [A smooth (enough) transition between the two solutions was performed at $x<-100$, corresponding to energies well above the cutoff determined by good matching for the truncation, so this does not affect the physics.]

\section{APPENDIX C: GEL'FAND-DIKII POLYNOMIALS}

In case they are needed, here are some of the higherorder Gel'Fand-Dikii polynomials, normalized such that the coefficient of the pure $u^{k}$ term is unity. In the following equation, a prime denotes an $x$ derivative times a factor of $\hbar$. For high numbers of derivatives, instead, a notation $u^{(n)}$ is used for $n$ primes. The first five are listed here:

$$
\begin{aligned}
\tilde{R}_{1}[u]= & u, \\
\tilde{R}_{2}[u]= & u^{2}-\frac{1}{3} u^{\prime \prime}, \\
\tilde{R}_{3}[u]= & u^{3}-\frac{1}{2}\left(u^{\prime}\right)^{2}-u u^{\prime \prime}+\frac{1}{10} u^{\prime \prime \prime \prime}, \\
\tilde{R}_{4}[u]= & u^{4}-2 u\left(u^{\prime}\right)^{2}-2 u^{2} u^{\prime \prime}+\frac{4}{5} u^{\prime} u^{\prime \prime \prime}+\frac{3}{5}\left(u^{\prime \prime}\right)^{2} \\
& +\frac{2}{5} u u^{\prime \prime \prime \prime}-\frac{1}{35} u^{(6)}, \\
\tilde{R}_{5}[u]= & u^{5}-5 u^{2}\left(u^{\prime}\right)^{2}-\frac{10}{3} u^{3} u^{\prime \prime}+\frac{11}{3} u^{\prime \prime}\left(u^{\prime}\right)^{2} \\
& +3 u\left(u^{\prime \prime}\right)^{2}+4 u u^{\prime} u^{\prime \prime \prime}+u^{2} u^{\prime \prime \prime \prime}-\frac{23}{42}\left(u^{\prime \prime \prime}\right)^{2} \\
& -\frac{19}{21} u^{\prime \prime \prime \prime} u^{\prime \prime}-\frac{3}{7} u^{(5)} u^{\prime}-\frac{1}{7} u^{(6)} u+\frac{1}{126} u^{(8)} .
\end{aligned}
$$

It will transpire that $\tilde{R}_{6}[u]$ and $\tilde{R}_{7}[u]$ will be needed as well, in order to get the required level of accuracy for the quantities computed in this paper. They are rather lengthy quantities, so it is not clear if there is much value in listing them here. Instead, they (and higher-order ones) can be easily computed using the recursion relation

$$
C_{k} \tilde{R}_{k+1}^{\prime}=\frac{\hbar^{3}}{4} \tilde{R}_{k}^{\prime \prime \prime}-u \hbar \tilde{R}_{k}^{\prime}-\frac{\hbar}{2} u^{\prime} \tilde{R}_{k}
$$

and the requirement that they vanish when $u$ does. $C_{k}$ is chosen to normalize the pure $u^{k}$ term to unity.

More useful, therefore, are the following three lines of Maple code, which can be iterated, with obvious adjustments, to get to arbitrarily high order:

$$
\begin{aligned}
>\mathrm{R}[1]:= & \mathrm{u}(\mathrm{x}) \\
>\mathrm{DR}[2]:= & (1 / 4) * \mathrm{~h}^{3} *(\operatorname{diff}(\mathrm{R}[1], \mathrm{x} \$ 3)) \\
& -\mathrm{u}(\mathrm{x}) * \mathrm{~h} *(\operatorname{diff}(\mathrm{R}[1], \mathrm{x})) \\
& -(1 / 2) * \mathrm{~h} *(\operatorname{diff}(\mathrm{u}[\mathrm{x}], \mathrm{x})) * \mathrm{R}[1] ; \\
>\mathrm{R}[2]:= & \operatorname{simplify}(-4 * \operatorname{integrate}(\mathrm{DR}[2], \mathrm{x}) /(3 * \mathrm{~h})) ;
\end{aligned}
$$

[1] R. Jackiw, Nucl. Phys. B252, 343 (1985).

[2] C. Teitelboim, Phys. Lett. 126B, 41 (1983).

[3] C. V. Johnson, Phys. Rev. D 101, 106023 (2020).
[4] C. V. Johnson, Preceding paper, Phys. Rev. D 103, 046012 (2021).

[5] P. Saad, S. H. Shenker, and D. Stanford, arXiv:1903.11115. 
[6] D. Stanford and E. Witten, arXiv:1907.03363.

[7] T. Guhr, A. Muller-Groeling, and H. A. Weidenmuller, Phys. Rep. 299, 189 (1998).

[8] J. Liu, Phys. Rev. D 98, 086026 (2018).

[9] K. Okuyama and K. Sakai, J. High Energy Phys. 08 (2020) 126.

[10] A. Blommaert, T. G. Mertens, and H. Verschelde, arXiv: 1911.11603.

[11] A. Altland and M. R. Zirnbauer, Phys. Rev. B 55, 1142 (1997).

[12] K. Okuyama and K. Sakai, J. High Energy Phys. 01 (2020) 156.

[13] P. Betzios and O. Papadoulaki, J. High Energy Phys. 09 (2020) 125.

[14] T. G. Mertens and G. J. Turiaci, arXiv:2006.07072.

[15] G. Sárosi, Proc. Sci., Modave2017 (2018) 001 [arXiv:1711 .08482].

[16] K. Jensen, Phys. Rev. Lett. 117, 111601 (2016).

[17] J. Maldacena and D. Stanford, Phys. Rev. D 94, 106002 (2016).

[18] J. Maldacena, D. Stanford, and Z. Yang, Prog. Theor. Exp. Phys. (2016), 12C104.

[19] J. Engelsöy, T. G. Mertens, and H. Verlinde, J. High Energy Phys. 07 (2016) 139.

[20] D. Stanford and E. Witten, J. High Energy Phys. 10 (2017) 008.

[21] A. Achucarro and M. E. Ortiz, Phys. Rev. D 48, 3600 (1993).

[22] A. Fabbri, D. Navarro, and J. Navarro-Salas, Nucl. Phys. B595, 381 (2001).

[23] P. Nayak, A. Shukla, R. M. Soni, S. P. Trivedi, and V. Vishal, J. High Energy Phys. 09 (2018) 048.

[24] K. S. Kolekar and K. Narayan, Phys. Rev. D 98, 046012 (2018).

[25] A. Ghosh, H. Maxfield, and G. J. Turiaci, J. High Energy Phys. 05 (2020) 104.

[26] A. Almheiri and J. Polchinski, J. High Energy Phys. 11 (2015) 014.

[27] S. Sachdev and J. Ye, Phys. Rev. Lett. 70, 3339 (1993).

[28] A. Kitaev, KITP seminars, April 7th and May 27th (2015).

[29] K. Papadodimas and S. Raju, Phys. Rev. Lett. 115, 211601 (2015).

[30] J. M. Maldacena, J. High Energy Phys. 04 (2003) 021.

[31] J. S. Cotler, G. Gur-Ari, M. Hanada, J. Polchinski, P. Saad, S. H. Shenker, D. Stanford, A. Streicher, and M. Tezuka, J. High Energy Phys. 05 (2017) 118; 09 (2018) 002(E).

[32] A. M. García-García and J. J. M. Verbaarschot, Phys. Rev. D 94, 126010 (2016).

[33] E. Brezin and V. A. Kazakov, Phys. Lett. B 236, 144 (1990).

[34] M. R. Douglas and S. H. Shenker, Nucl. Phys. B335, 635 (1990).

[35] D. J. Gross and A. A. Migdal, Phys. Rev. Lett. 64, 127 (1990).

[36] D. J. Gross and A. A. Migdal, Nucl. Phys. B340, 333 (1990).

[37] W. Fu, D. Gaiotto, J. Maldacena, and S. Sachdev, Phys. Rev. D 95, 026009 (2017); 95, 069904(A) (2017).

[38] T. Li, J. Liu, Y. Xin, and Y. Zhou, J. High Energy Phys. 06 (2017) 111.

[39] T. Kanazawa and T. Wettig, J. High Energy Phys. 09 (2017) 050.

[40] F. Sun and J. Ye, Phys. Rev. Lett. 124, 244101 (2020).
[41] S. Forste and I. Golla, Phys. Lett. B 771, 157 (2017).

[42] J. Murugan, D. Stanford, and E. Witten, J. High Energy Phys. 08 (2017) 146.

[43] P. Saad, S. H. Shenker, and D. Stanford, arXiv:1806.06840.

[44] P. H. Ginsparg and G. W. Moore, in Theoretical Advanced Study Institute (TASI 92): From Black Holes and Strings to Particles (1993), pp. 277-469 [arXiv:hep-th/9304011].

[45] N. Seiberg and D. Shih, C. R. Phys. 6, 165 (2005).

[46] T. Banks, M. R. Douglas, N. Seiberg, and S. H. Shenker, Phys. Lett. B 238, 279 (1990).

[47] M. R. Douglas, Phys. Lett. B 238, 176 (1990).

[48] I. R. Klebanov, J. M. Maldacena, and N. Seiberg, Commun. Math. Phys. 252, 275 (2004).

[49] T. R. Morris, Nucl. Phys. B356, 703 (1991).

[50] S. Dalley, C. V. Johnson, and T. Morris, Nucl. Phys. B368, 625 (1992).

[51] S. Dalley, C. V. Johnson, T. R. Morris, and A. Watterstam, Mod. Phys. Lett. A 07, 2753 (1992).

[52] S. Dalley, C. V. Johnson, and T. Morris, Nucl. Phys. B368, 655 (1992).

[53] S. Dalley, C. V. Johnson, and T. Morris, Nucl. Phys. B, Proc. Suppl. 25A, 87 (1992).

[54] I. M. Gel'fand and L. A. Dikii, Russ. Math. Surv. 30, 77 (1975).

[55] V. A. Kazakov, Mod. Phys. Lett. A 04, 2125 (1989).

[56] I. K. Kostov, Phys. Lett. B 238, 181 (1990).

[57] J. E. Carlisle, C. V. Johnson, and J. S. Pennington, J. Phys. A 40, 12451 (2007).

[58] M. Pillai, J. Goglio, and T. G. Walker, Am. J. Phys. 80, 1017 (2012).

[59] S. H. Shenker, The strength of nonperturbative effects in string theory, in Random Surfaces and Quantum Gravity, NATO ASI Series Vol. 262 (Springer, 1991), pp. 191-200, https://doi.org/10.1007/978-1-4615-3772-4.

[60] A. B. Zamolodchikov and A. B. Zamolodchikov, arXiv:hepth/0101152.

[61] C. V. Johnson, J. High Energy Phys. 12 (2004) 072.

[62] J. Maldacena and X.-L. Qi, arXiv:1804.00491.

[63] N. Hunter-Jones and J. Liu, J. High Energy Phys. 05 (2018) 202.

[64] C. Lau, C.-T. Ma, J. Murugan, and M. Tezuka, arXiv: 2003.05401

[65] D. J. Gross, J. Kruthoff, A. Rolph, and E. Shaghoulian, Phys. Rev. D 102, 046019 (2020).

[66] D. J. Gross, J. Kruthoff, A. Rolph, and E. Shaghoulian, Phys. Rev. D 101, 026011 (2020).

[67] L. V. Iliesiu, J. Kruthoff, G. J. Turiaci, and H. Verlinde, arXiv:2004.07242.

[68] D. Stanford and Z. Yang, arXiv:2004.08005.

[69] F. David, Mod. Phys. Lett. A 05, 1019 (1990).

[70] M. R. Douglas, N. Seiberg, and S. H. Shenker, Phys. Lett. B 244, 381 (1990).

[71] S. Dalley, Phys. Lett. B 253, 292 (1991).

[72] G. W. Moore, Commun. Math. Phys. 133, 261 (1990).

[73] G. W. Moore, Prog. Theor. Phys. Suppl. 102, 255 (1990).

[74] E. Witten, arXiv:2006.03494.

[75] H. Maxfield and G. J. Turiaci, arXiv:2006.11317.

[76] A. Okounkov, arXiv:math/0101201.

[77] C. V. Johnson, Ph. D. thesis, Southampton University, UK, 1992.

[78] C. V. Johnson, T. R. Morris, and A. Watterstam, Phys. Lett. B 291, 11 (1992). 